\title{
A Programmer's Reference Guide to FDMS File Formats
}

Rebecca W. Portier

Building and Fire Research Laboratory

Gaithersburg, Maryland 20899

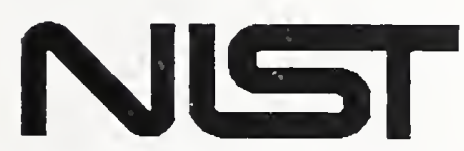

United States Department of Commerce

Technology Administration

National Institute of Standards and Technology 



\section{A Programmer's Reference Guide to FDMS File Formats}

Rebecca W. Portier

April 1993

Building and Fire Research Laboratory

National Institute of Standards and Technology

Gaithersburg, MD 20899

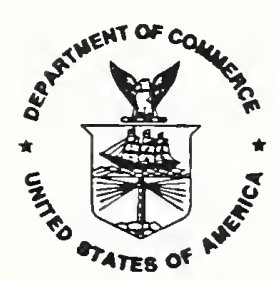

U.S. Department of Commerce

Ronald H. Brown, Secretary

National Institute of Standards and Technology

Raymond Kammer, Acting Director 



\section{CONTENTS}

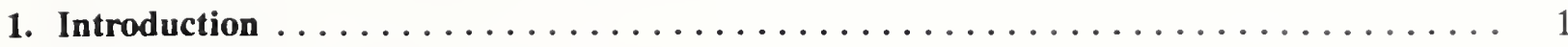

2. FDMS Beta Version File Formats $\ldots \ldots \ldots \ldots \ldots \ldots \ldots \ldots \ldots \ldots \ldots \ldots \ldots \ldots \ldots \ldots$

2.1 CONE

$\begin{array}{ll}2.2 \text { FURN } & 6\end{array}$

2.3 FRESIST $r$

\begin{tabular}{l}
2.4 ORGANISE \\
\hline
\end{tabular}

2.5 PEOPLE $\quad 9$

2.6 INSTRUM 10

2.7 CALIB 10

2.8 PRODUCT 11

3. Future Modifications to FDMS Beta Version File Formats . . . . . . . . . . . . . . . . 11

3.1 CONE 12

3.2 FURN 14

$\begin{array}{ll}3.3 \text { LIFT } & 16\end{array}$

$\begin{array}{llr}3.4 & \text { ROOM } & 18\end{array}$

$\begin{array}{ll}3.5 & \text { FRESIST }\end{array}$

3.6 ORGANISE $\quad 21$

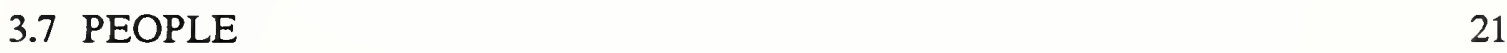

3.8 INSTRUM $\quad 22$

$\begin{array}{ll}3.9 \text { CALIB } & 22\end{array}$

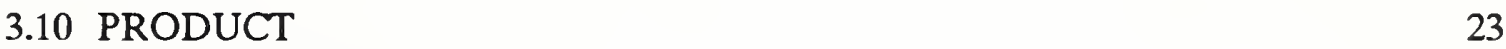

4. FDMS Beta Version Raw Data File . . . . . . . . . . . . . . . . . . . . . . . 23

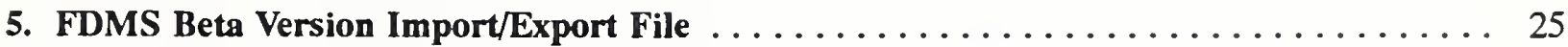

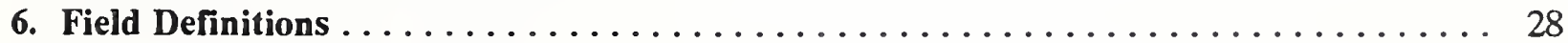

7. References $\ldots \ldots \ldots \ldots \ldots \ldots \ldots \ldots \ldots \ldots \ldots \ldots \ldots \ldots \ldots \ldots \ldots \ldots$ 



\title{
A Programmer's Reference Guide to FDMS File Formats
}

\author{
Rebecca W. Portier \\ Building and Fire Research Laboratory \\ National Institute of Standards and Technology
}

\begin{abstract}
Fire Data Management System, FDMS, is a computer database specifically designed to store and retrieve fire test results. This guide provides detailed descriptions of the current, beta version, file formats as well as revisions planned for the immediate future.
\end{abstract}

Key words: computer database; Cone Calorimeter; export; Furniture Calorimeter; import; LIFT apparatus; room/corner test; scalar data; vector data

\section{Introduction}

A unified method of accessing data is crucial to both experimental and modeling efforts in the development of the science of fire. FDMS, the Fire Data Management System $[1]^{1}$, is a computer database for organizing and presenting fire data obtained from small-scale and large-scale tests as well as fire simulation programs. By storing available fire test values in a common format, this data is readily available to computer models, plotting programs, and report generators.

The goal for FDMS is to provide a centralized database of test values generated from a variety of sources within the fire community. Such a database could be accessed through communications networks providing all participants with immediate access to new results. The FDMS concept should not be limited by computer platforms, computer languages, or data inflexibilities. Development of this centralized FDMS database involves four stages and the release of two versions of the software program.

The initial stage of development provides a beta version software program which can be used transitionally to store results and to exchange test values between participants. A beta version of the FDMS software currently exists[2] which provides this functionality. One goal of this FDMS beta version is the generation of feedback from the user community. The later, centralized version of the FDMS software must provide an open file design that will easily accommodate future test apparatus formats. All file formats and program functionality provided in the beta version will be supported in the later version along with appropriate user

1 Numbers in brackets refer to literature references listed in Section 7 at the end of this report. 
recommended additions. Consequently, user response is critical to the successful design of the centralized FDMS database.

This reference guide is intended to address the second stage of development by providing details of the FDMS beta version internal file formats. This includes database files as well as import and export formats. These formats are detailed to assist model developers in accessing test data in the FDMS beta version and in verifying that all data required by their models is available. The later, centralized version of FDMS will include all test apparatus available in the beta version, all test apparatus detailed in the original design of FDMS[3], and appropriate recommended additions and modifications. Response from model developers is critical to the successful design of the centralized FDMS database file formats.

A second version of the FDMS software will be developed and released in the third stage of development by incorporating feedback from the FDMS beta version users and model developers. The new version must minimally support the functionality and data details of the beta version and provide a new user interface that is independent of computer platform.

In the final stage, the centralized database will be generated using data provided from each of the existing individual databases. Once the database has been generated, access through communication networks will be provided. Data from the central database can be accessed at user locations or downloaded for access in individual FDMS software programs.

The results of the file format evaluations for the beta version of FDMS have been divided into four sections in this reference. Section 2 covers database file formats as they presently exist. Section 3 presents additional modifications which must be made to the FDMS beta version in order to accommodate existing fire models. The modifications recommended in Section 3 are results of an early evaluation and have not been finalized. Feedback to these suggested modifications is encouraged. Sections 4 and 5 provide details for the import formats supported by the beta version of FDMS.

This reference is not intended to provide instructions on the use of the FDMS beta version. Operation details are available in the FDMS user's guide and technical documentation $[2,3]$. Some experience with computer databases and database concepts is assumed throughout the remainder of this reference guide.

\section{FDMS Beta Version File Formats}

The FDMS beta version software stores scalar and vector fire test values. Each scalar data field allows entry of at most one value for each test run. Scalar data is stored within the FDMS database. Examples of scalar data include the test operator's name, the date of the test, and the time to ignition of the sample. Vector data is stored in external ASCII files as columns of numbers. Each value in the column is dependent on a corresponding value within a second set of vector data. The rate of heat release is an example of vector data. The 
corresponding set of vector values in this case would be the times at which the rate of heat release was measured and recorded.

Database files for the beta version of FDMS can be categorized as either main test apparatus files or secondary files providing supplementary information. Examples of supplementary information include participating organizations, personnel contacts, and product manufacturing details. Data tables have been defined within the FDMS system for a large number of commonly used fire tests. The beta version software fully implements only those for the Cone Calorimeter, Furniture Calorimeter, and the fire-resistance table[4]. This reference section provides technical details for the internal format of these main test files and all secondary files.

Fields within the database files are categorized according to the type of values entered. String\$ fields allow entry of numbers, letters, and special characters from a standard computer keyboard. UCase\$ and Numeric\$ are special types of String\$ fields. UCase\$ converts all letters entered to upper case before storing while Numeric $\$$ allows only numbers and numeric symbols. Single! fields store single-precision floating point numbers. Integer\% stores integer values which may be updated later by arithmetic calculations. Date fields store formatted date values as the month, day, year format (MM/DD/YY). Logical fields allow entry for one of two possible values. Examples of Logical field values are $\mathrm{Y}$ or $\mathrm{N}$ (yes or no), and $\mathrm{H}$ or $\mathrm{V}$ (horizontal or vertical). Choice fields are similar to logical fields except that values are selected from a predefined set. For an example of a Choice field, refer to the IGNTYPE field in Section 6. Relational fields allow entry of any value found in a corresponding field of a secondary file. The concept is similar to the Choice field but is limited to existing values entered in the related file by the FDMS user.

The internal structure of each file is presented in this section by comparing the existing format to the original design specifications. Any variations are highlighted. The original specifications can be found in the FDMS technical documentation $[2,3]$. Each format specification details file offsets to provide model developers direct access to the stored values without restricting development to the language and database engine used in the existing version of FDMS. Variations in screen format or display format are included so that developers generating import and export files can support the same numeric precision. Highlighted field name changes indicate the need for corresponding adjustments in files generated in the FDMS import format. The field name as it exists in the existing beta version is highlighted in the field name column with the previous name displayed to the right. New fields are indicated with a * NEW

A field description section is provided in Section 6 to minimize the need to refer to the original FDMS document. The field definitions are identical in both documents.

REMINDER: Highlighting throughout this section indicates modifications to the original FDMS file format that have been implemented in the beta version of FDMS. 


\section{$2.1 \mathrm{CONE}$}

The HF field originally allocated to hydrogen fluoride measurements was reallocated to total unburned hydrocarbons, FUT, to conform to current usage in laboratories.

CONE: 95 fields, record length $=929$

\begin{tabular}{|c|c|c|c|c|c|c|}
\hline Id & File & Field & & Screen & \multirow{2}{*}{\multicolumn{2}{|c|}{$\begin{array}{l}\text { Related/Choice } \\
\text { File Field非 }\end{array}$}} \\
\hline 非 & Offset & Name & Type & Format & & \\
\hline 0 & 1 & Deleted & if "!" & & & \\
\hline 1 & 2 & LABID & Relational & o & ORGANISE & 1 \\
\hline 2 & 10 & FILE & strings & 8 & & \\
\hline 3 & 18 & RECEIVED & Date & 10 & & \\
\hline 4 & 20 & PRIVATE & Choice & $10 \%$ & Graul & 絊 \\
\hline 5 & 22 & ADMIN & String\$ & 8. & & \\
\hline 6 & 30 & TESTDATE & Date & 10 & & \\
\hline 7 & 32 & REPDATE & Date & 10 & NeOex & 3 \\
\hline 8 & 34 & OPERATOR & Relational & $40 \%$ & PEOPLE & 3 \\
\hline 9 & 74 & OPERID & Relational & 8 & PEOPLE & 1 \\
\hline 10 & 82 & OFFICER & Relational & 100 & PEOPLE & 3 \\
\hline 11 & 122 & OFFID & Relational & 8 & PEOPLE & 1 \\
\hline 12 & 130 & SPONSOR & Relational & 50 & ORGANISE & 3 \\
\hline 13 & 180 & SPONID & Relational & 7 & ORGANISE & 1 \\
\hline 14 & 187 & SPONCONT & Relational & $40 \%$ & PEOPLE & 3 \\
\hline 15 & 227 & SPCONTID & Relational & 7 & PEOPLE & 1 \\
\hline 16 & 234 & PRODUCT1 & Relational & $50 \%$ & PRODUCT & 3 \\
\hline 17 & 284 & PRODIDI & Relational & 7 & PRODUCT & 1 \\
\hline 18 & 291 & PRODUCT2 & Relational & $50 \%$ & PRODUCT & 3 \\
\hline 19 & 341 & PRODID2 & Relational & 7 & PRODUCT & 1 \\
\hline 20 & 348 & FLUX & Single! & 5.1 & & \\
\hline 21 & 352 & FLOW & Single! & 4.1 & & \\
\hline 22 & 356 & THICK & Single! & 9.6 & & \\
\hline 23 & 360 & AREA & Single! & 6. & & \\
\hline 24 & 364 & $\mathrm{C}$ & Single! & 9.6 & & \\
\hline 25 & 368 & $\mathrm{E}$ & Single! & 8.8 .5 & & \\
\hline 26 & 372 & OXYGEN & Single! & 5.2 & & \\
\hline 27 & 376 & RHCOND & Single! & 4.1 & & \\
\hline 28 & 380 & TEMPCOND & Single! & 5.1 & & \\
\hline 29 & 384 & RHTEST & Single! & $4 \times$ & & \\
\hline 30 & 388 & TEMPTEST & Single! & 5. 1 & & \\
\hline 31 & 392 & ORIENT & Logical & 1 & $\mathrm{H} \mathrm{V}$ & \\
\hline 32 & 393 & PILOT & Logical & 1 & $\mathrm{YN}$ & \\
\hline 33 & 394 & GRID & Logical & 1 & $\mathrm{~N} \mathrm{Y}$ & \\
\hline 34 & 395 & FRAME & Logical & 1 & Y N & \\
\hline 35 & 396 & ASCARITE & - Logical & 1 & $\mathrm{Y} \mathrm{N}$ & \\
\hline 36 & 397 & MSTRNO & Strungs: & 4 & INSTRUM & 1 \\
\hline 37 & 401 & SCANS & Integer\% & 46 & & \\
\hline 38 & 403 & INTERVAL & Integer\% & 2 & & \\
\hline 39 & 405 & COMMENT1 & String\$ & 60 & & \\
\hline
\end{tabular}

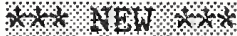

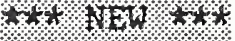

$4 .+14.4$.

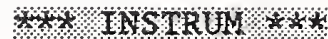




\begin{tabular}{|c|c|c|c|c|}
\hline 40 & 465 & COMMENT 2 & String\$ & 60 \\
\hline 41 & 525 & COMMENT3 & String\$ & 60 \\
\hline 42 & 585 & COMMENT4 & String\$ & 60 \\
\hline 43 & 645 & COMMENT5 & String\$ & 60 \\
\hline 44 & 705 & MASSI & Single! & 7 \\
\hline 45 & 709 & MASSF & Single! & 7.1 \\
\hline 46 & 713 & MASSLOSS & Single! & 7.2 \\
\hline 47 & 717 & TIGN & Integer\% & 5 \\
\hline 48 & 719 & FLAMEOUT & Integer\% & 5 \\
\hline 49 & 721 & MAXTIME & Integer\% & 5 \\
\hline 50 & 723 & MAXQDOT & Single! & 9.1 \\
\hline 51 & 727 & MAXMDOT & Single! & 9.2 \\
\hline 52 & 731 & MAXSIGMA & Single! & 9.2 \\
\hline 53 & 735 & TOTLHEAT & Single! & 9.2 \\
\hline 54 & 739 & AVGQDOT & Single! & 9.2 \\
\hline 55 & 743 & AVGMDOT & Single! & 9.2 \\
\hline 56 & 747 & AVGHC & Single! & 9.2 \\
\hline 57 & 751 & AVS IGMA & Single! & 9.2 \\
\hline 58 & 755 & AVGCO2 & Single! & 9.5 \\
\hline 59 & 759 & AVGCO & Single! & 9.5 \\
\hline 60 & 763 & AVGH2O & Single! & 9.5 \\
\hline 61 & 767 & QDOT60 & Single! & 9.2 \\
\hline 62 & 771 & MDOT60 & Single! & 9.2 \\
\hline 63 & 775 & HC60 & Single! & 9.2 \\
\hline 64 & 779 & SIGMA60 & Single! & 9.2 \\
\hline 65 & 783 & $\mathrm{CO} 260$ & Single! & 9.5 \\
\hline 66 & 787 & $\mathrm{CO} 60$ & Single! & 9.8 \\
\hline 67 & 791 & H2060 & Single! & 95 \\
\hline 68 & 795 & QDOT180 & Single! & 9 江 \\
\hline 69 & 799 & MDOT180 & Single! & 9.2 \\
\hline 70 & 803 & $\mathrm{HCl} 80$ & Single! & 9.2 \\
\hline 71 & 807 & SIGMA180 & Single! & 9.2 \\
\hline 72 & 811 & $\mathrm{CO} 2180$ & Single! & 9.5 \\
\hline 73 & 815 & Col80 & Single! & 69 \\
\hline 74 & 819 & $\mathrm{H} 20180$ & Single! & 9.9 \\
\hline 75 & 823 & QDOT300 & Single! & 9.2 \\
\hline 76 & 827 & MDOT300 & Single! & 9.2 \\
\hline 77 & 831 & HC 300 & Single! & 9.2 \\
\hline 78 & 835 & SIGMA 300 & Single! & 9,2 \\
\hline 79 & 839 & $\mathrm{CO} 2300$ & Single! & 9.5 \\
\hline 80 & 843 & $\mathrm{co3} 00$ & Single! & 9.5 \\
\hline 81 & 847 & H2O300 & Single! & 9.5 \\
\hline 82 & 851 & SOOT & Single! & 9.1 \\
\hline 83 & 855 & HCL & Single! & (6) \\
\hline 84 & 859 & $\mathrm{HCN}$ & Single! & 9.5 \\
\hline 85 & 863 & HBR & Single! & 9.5 \\
\hline 86 & 867 & UUH & Single! & 9.5 \\
\hline 87 & 871 & USER IS & String & 10 \\
\hline 88 & 881 & USERS 2 & String\$ & 10 \\
\hline
\end{tabular}

*** $\mathrm{NEW} * * \times$

*** NEW $+*$ *

$* * x$ AVGSTGM $* *$ X
$* * x$.

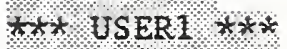
$* * *, \operatorname{tsER2}, * * t$ 


\begin{tabular}{|c|c|c|c|}
\hline 891 & USER 35 & String\$ & 10 \\
\hline 901 & CSERNUMI & Single! & $10 \%$ \\
\hline 905 & US ERNUM2 & Single! & 10.2 \\
\hline 909 & USERNUMS & Single! & 10.3 \\
\hline 913 & VERS ION & Nune r 4 cs & 5 \\
\hline 918 & TEST & I tumer ICS & 5 \\
\hline 923 & ZNUMBER & Nutue r l c s & 7 \\
\hline
\end{tabular}

\%) USER 3 \% \&

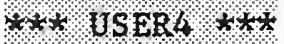
**V. USERS . $7 * \%$

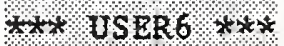

\subsection{FURN}

FURN : 92 fields, record length $=951$

\begin{tabular}{|c|c|c|c|c|c|c|}
\hline $\begin{array}{l}\text { Fld } \\
\text { 非 }\end{array}$ & $\begin{array}{c}\text { File } \\
\text { offset }\end{array}$ & $\begin{array}{l}\text { Field } \\
\text { Name }\end{array}$ & Type & $\begin{array}{l}\text { Screen } \\
\text { Format }\end{array}$ & \multirow{2}{*}{\multicolumn{2}{|c|}{$\begin{array}{l}\text { Related/Choice } \\
\text { File Field作 }\end{array}$}} \\
\hline 0 & $\frac{1}{1}$ & Deleted & if "!" & & & \\
\hline 1 & 2 & LABID & Relational & 8 & ORGANISE & 1 \\
\hline 2 & 10 & FILE & String\$ & 8 & & \\
\hline 3 & 18 & RECEIVED & Strings & 10 & & \\
\hline 4 & 28 & PRIVATE & Choice & $10 \%$ & - OLRCIUL & 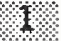 \\
\hline 5 & 30 & ADMIN & String\$ & 8. & & \\
\hline 6 & 38 & TESTDATE & strings. & 10 & & \\
\hline 7 & 48 & REPDATE & stelngs & 10 & BeOPII & 3 \\
\hline 8 & 58 & OPERATOR & Relational & 40 & PEOPLE & 3 \\
\hline 9 & 98 & OPERID & Relational & 8 & PEOPLE & 1 \\
\hline 10 & 106 & OFFICER & Relational & 40 & PEOPLE & 3 \\
\hline 11 & 146 & OFFID & Relational & 8 & PEOPLE & 1 \\
\hline 12 & 154 & SPONSOR & Relational & 50 & ORGANISE & 3 \\
\hline 13 & 204 & SPONID & Relational & 7 & ORGANISE & 1 \\
\hline 14 & 211 & SPONCONT & Relational & $40 \%$ & PEOPLE & 3 \\
\hline 15 & 251 & SPCONTID & Relational & 7 & PEOPLE & 1 \\
\hline 16 & 258 & PRODUCT1 & Relational & 80 & PRODUCT & 3 \\
\hline 17 & 308 & PRODIDI & Relational & 7 & PRODUCT & 1 \\
\hline 18 & 315 & PRODUCT2 & Relational & 50 & PRODUCT & 3 \\
\hline 19 & 365 & PRODID2 & Relational & 7 & PRODUCT & 1 \\
\hline 20 & 372 & FLUX & Single! & 5.1 & & \\
\hline 21 & 376 & FLOW & Single! & 4.1 & & \\
\hline 22 & 380 & THICK & Single! & 96 & & \\
\hline 23 & 384 & AREA & Single! & 9.6 & & \\
\hline 24 & 388 & C & Single! & 9.6 & & \\
\hline 25 & 392 & $E$ & Single! & 8.5 & & \\
\hline 26 & 396 & OXYGEN & Single! & 5.2 & & \\
\hline 27 & 400 & RHCOND & Single! & 4. & & \\
\hline 28 & 404 & TEMPCOND & Single! & 5.1 & & \\
\hline 29 & 408 & RHTEST & Single! & 6. 1 & & \\
\hline 30 & 412 & TEMPTEST & Single! & 5. & & \\
\hline 31 & 416 & MGNTPEP & Choice & 30: & IGNOMOR & 1 Ix \\
\hline 32 & 418 & ASCARITE & Logical & 1 & Y N & \\
\hline 33 & 419 & INSTRNO & Strings: & \% & INSTRUM & 1 \\
\hline 34 & 423 & SCANS & Integer\% & 㱍 & & \\
\hline
\end{tabular}

* V VEW *

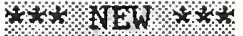

WEW $+2+1 \mathrm{NEW}+\mathrm{tr}$ WHONOR $\%$ WHSTHM + H. 


\begin{tabular}{|c|c|c|c|c|}
\hline 35 & 425 & INTERVAL & Integer\% & 2 \\
\hline 36 & 427 & COMMENT1 & Strings & 60 \\
\hline 37 & 487 & COMMENT2 & String\$ & 60 \\
\hline 38 & 547 & COMMENT3 & String\$ & 60 \\
\hline 39 & 607 & COMMENT4 & String\$ & 60 \\
\hline 40 & 667 & COMMENT5 & string\$ & 60 \\
\hline 41 & 727 & MASSI & Single! & 1 \\
\hline 42 & 731 & MASSF & Single! & 7 : \\
\hline 43 & 735 & MASSLOSS & Single! & 8.3 \\
\hline 44 & 739 & TIGN & Integer\% & 5 \\
\hline 45 & 741 & FLAMEOUT & Integer\% & 5 \\
\hline 46 & 743 & MAXTIME & Integer\% & 5 \\
\hline 47 & 745 & MAXQDOT & Single! & 9.1 \\
\hline 48 & 749 & MAXMDOT & Single! & 9.1 \\
\hline 49 & 753 & MAXSIGMA & Single! & 9.2 \\
\hline 50 & 757 & TOTLHEAT & Single! & 10.1 \\
\hline 51 & 761 & AVGQDOT & Single! & 9.1 \\
\hline 52 & 765 & AVGMDOT & Single! & 9.1 \\
\hline 53 & 769 & AVGHC & Single! & 9.2 \\
\hline 54 & 773 & AYSTGMA & Single! & 9.2 \\
\hline 55 & 777 & AVGCO2 & Single! & 9.5 \\
\hline 56 & 781 & AVGCO & Single! & 9.5 \\
\hline 57 & 785 & AVGH2O & Single! & 9.3 \\
\hline 58 & 789 & QDOT60 & Single! & 9.1 \\
\hline 59 & 793 & MDOT60 & Single! & 9.1 \\
\hline 60 & 797 & HC60 & Single! & 9.2 \\
\hline 61 & 801 & SIGMA60 & Single! & 9.2 \\
\hline 62 & 805 & $\mathrm{CO} 260$ & Single! & 9.5 \\
\hline 63 & 809 & $\mathrm{CO} 60$ & Single! & 9.5 \\
\hline 64 & 813 & $\mathrm{H} 2060$ & Single! & 9.5 \\
\hline 65 & 817 & QDOT180 & Single! & 9.1 \\
\hline 66 & 821 & MDOT180 & Single! & 9.1 \\
\hline 67 & 825 & $\mathrm{HCl} 80$ & Single! & 9.2 \\
\hline 68 & 829 & SIGMA180 & Single! & 9.2 \\
\hline 69 & 833 & $\mathrm{CO} 2180$ & Single! & 9.5 \\
\hline 70 & 837 & C0180 & Single! & 9.5 \\
\hline 71 & 841 & H2O180 & Single! & 9.5 \\
\hline 72 & 845 & QDOT300 & Single! & 9.1 \\
\hline 73 & 849 & MDOT300 & Single! & 9.1 \\
\hline 74 & 853 & HC 300 & Single! & 9.2 \\
\hline 75 & 857 & SIGMA 300 & Single! & 9.2 \\
\hline 76 & 861 & $\mathrm{CO} 2300$ & Single! & 9.5 \\
\hline 77 & 865 & $\mathrm{CO} 300$ & Single! & 9.5 \\
\hline 78 & 869 & $\mathrm{H} 20300$ & Single! & $9 ., 5$ \\
\hline 79 & 873 & SOOT & Single! & 9.5 \\
\hline 80 & 877 & HCL & Single! & 9.5 \\
\hline 81 & 881 & $\mathrm{HCN}$ & Single! & 9.5 \\
\hline 82 & 885 & HBR & Single! & 9.5 \\
\hline 83 & 889 & $\mathrm{HF}$ & Single! & 9.5 \\
\hline
\end{tabular}

$x+*$ N $x+x$

$* * *$ StMQ $* * *$

$* *$ AVGSIGMA $* * *$

$1+* \mathrm{NETH}+\mathrm{H}$

$* * *$ NEW $* * t$

$* *+\mathrm{NEW} * x+$

$*+x$ NEW $*+* x$

$t+t$, ten $t+t$

$t * t . \mathrm{nEW} * t+t$

$*+*$ N NEW $t+*$

$* *+\mathrm{NEW} * *+$

$7+*$ NEW $*+*$

$*+*$ NEW $6+t$

$* * *$ NEW $* * *$

$* x+\mathrm{NEW} * x+x$

$r+*$ NEW $*+*$

$* *+$ NEW $* * t$

$*+*$ V NEW $*+*$

$* *+* \mathrm{NEH} *+\cdots$

$6+2$ IEW $t+t$

$x+t$ VEW $* x+$

$* *+$ VEW $* x+*$

$* * *$ iten $* *+*$

$* x+$ VEW $t+*$ 


\begin{tabular}{|c|c|c|c|c|}
\hline 84 & 893 & USER IS & String\$ & 10 \\
\hline 85 & 903 & USERS 2 & Strings & 10 \\
\hline 86 & 913 & USER 35 & String\$ & 10 \\
\hline 87 & 923 & USERUUMUI & Single! & 10.2 \\
\hline 88 & 927 & GSERNUN2 & Single! & 10.2 \\
\hline 89 & 931 & GSERMUMS & Single! & 10.3 \\
\hline 90 & 935 & VERSION & Muner 1 < $S$ & 5 \\
\hline 91 & 940 & TEST & Numer 1 es & 5 \\
\hline 92 & 945 & ZNUMBER & Nume r $1 \mathrm{c} S$ & 7 \\
\hline
\end{tabular}

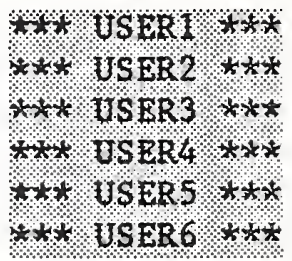

\subsection{FRESIST}

\begin{tabular}{|c|c|c|c|c|c|c|}
\hline $\begin{array}{l}\text { F1d } \\
\text { 韭 }\end{array}$ & $\begin{array}{c}\text { File } \\
\text { Offset }\end{array}$ & $\begin{array}{l}\text { Field } \\
\text { Name }\end{array}$ & Type & $\begin{array}{l}\text { Screen } \\
\text { Format }\end{array}$ & \multicolumn{2}{|c|}{$\begin{array}{l}\text { Related/Choice } \\
\text { File Field非 }\end{array}$} \\
\hline 0 & 1 & $\overline{\text { Deleted }}$ & if "!" & & & \\
\hline 1 & 2 & TESTCODE & Numeric\$ & 8 & & \\
\hline 2 & 10 & TDATE & Date & 8 & & \\
\hline 3 & 12 & SPONREF & Relational & 5 & ORGANISE & 1 \\
\hline 4 & 17 & SPONSOR & Relational & 50 & ORGANISE & 3 \\
\hline 5 & 67 & INSUTIME & Integer\% & 3 & & \\
\hline 6 & 69 & INSUCOND & String\$ & 22 & & \\
\hline 7 & 91 & INTETIME & Integer\% & 3 & & \\
\hline 8 & 93 & INTECOND & string\$ & 22 & & \\
\hline 9 & 115 & STABTIME & Integer\% & 3 & & \\
\hline 10 & 117 & STABCOND & String\$ & 22 & & \\
\hline 11 & 139 & TESTTYPE & UCase\$ & 2 & & \\
\hline 12 & 141 & PRODREF & Relational & 5 & PRODUCT & 1 \\
\hline 13 & 146 & FRDESC1 & String\$ & 55 & & \\
\hline 14 & 201 & FRDESC2 & String\$ & 55 & & \\
\hline 15 & 256 & FRDESC 3 & String\$ & 55 & & \\
\hline 16 & 311 & FRDESC4 & String\$ & 55 & & \\
\hline 17 & 366 & FRDESC5 & String\$ & 55 & & \\
\hline 18 & 421 & FRDESC6 & String\$ & 55 & & \\
\hline 19 & 476 & FRDESC7 & String\$ & 55 & & \\
\hline 20 & 531 & FRDESC 8 & StringS & 55 & & \\
\hline 21 & 586 & FRDESC 9 & String\$ & 55 & & \\
\hline
\end{tabular}




\subsection{ORGANISE}

ORGANISE: 14 fields, record length $=292$

\begin{tabular}{|c|c|c|c|c|c|}
\hline $\begin{array}{l}\text { FId } \\
\text { 非 }\end{array}$ & $\begin{array}{l}\text { File } \\
\text { offset }\end{array}$ & $\begin{array}{l}\text { Field } \\
\text { Name }\end{array}$ & Type & $\begin{array}{l}\text { Screen Related/Choice } \\
\text { Format File Field非 }\end{array}$ & \\
\hline 0 & 1 & $\overline{\text { Deleted }}$ & if "!n & & \\
\hline 1 & 2 & ORGID & Integert & 8 & \\
\hline 2 & 4 & CHEXORG & Numerics & 8 & $4 * *$ GHECKSUM $*+* *$ \\
\hline 3 & 12 & ORGANISE & String $\$$ & 50 & $* * t$ ORGAI $* * *$ \\
\hline 4 & 62 & DIVISION & Strings & 50 & \\
\hline 5 & 112 & ADDRESS 1 & Strings & 32 & \\
\hline 6 & 144 & ADDRESS2 & String\$ & 32 & \\
\hline 7 & 176 & CITY & Strings & 20 & \\
\hline 8 & 196 & REGION & String\$ & 12 & \\
\hline 9 & 208 & POSTCODE & UGases & 10 & \\
\hline 10 & 218 & COUNTRY & Strings & 20 & \\
\hline 11 & 238 & PHONE & Ucases & 15 & \\
\hline 12 & 253 & FAXIMILE & Numer les & 15 & FAX $1+$ F \\
\hline 13 & 268 & TELEX & UCases. & 15 & \\
\hline 14 & 283 & ORGDATE & Strings & 10 & $* * *$ DATE $* * *$ \\
\hline
\end{tabular}

\subsection{PEOPLE}

PEOPLE: 20 fields, record length $=435$

\begin{tabular}{|c|c|c|c|c|c|c|}
\hline $\begin{array}{l}\text { Fld } \\
\text { 非 }\end{array}$ & $\begin{array}{l}\text { File } \\
\text { Offset }\end{array}$ & $\begin{array}{l}\text { Field } \\
\text { Name }\end{array}$ & Type & $\begin{array}{l}\text { Screen Related/Choice } \\
\text { Format File Field非 }\end{array}$ & & \\
\hline 0 & 1 & $\overline{\text { Deleted }}$ & if "!" & & & \\
\hline 1 & 2 & PERSONTO & Integert & 7 7. & $t * t$ & PERSTD \% *X \\
\hline 2 & 4 & CHEKYPER & Rumerics & 8 & $1+*$ & GHECKSUM $++*$ \\
\hline 3 & 12 & NAMES & Strings & 40 & & \\
\hline 4 & 52 & FIRSTNAM & String\$ & 12 & & \\
\hline 5 & 64 & INITIAL & String\$ & 3 & & \\
\hline 6 & 67 & LASTNAME & String\$ & 20 & & \\
\hline 7 & 87 & ORGAVISE & String $\$$ & 50 & $x+x$ & ORGAN $t \times *$ \\
\hline 8 & 137 & DIVISION & Strings & 50 紧 & & \\
\hline 9 & 187 & ADDRESS1 & string\$ & 32 & & \\
\hline 10 & 219 & ADDRESS2 & String\$ & 32 & & \\
\hline 11 & 251 & CITY & Strings & 20 & & \\
\hline 12 & 271 & REGION & String\$ & 12 & & \\
\hline 13 & 283 & POSTCODE & Hosses & 10 & & \\
\hline 14 & 293 & COUNTRY & strings & 20 & & \\
\hline 15 & 313 & ADDINFO & String\$ & 50 & & \\
\hline 16 & 363 & PHONE & String\$ & 15 & & \\
\hline 17 & 378 & MORPHONE & strings & 18 & & \\
\hline 18 & 396 & FAX & String & 15 & & \\
\hline 19 & 411 & TELEX & String\$ & 15 . & & \\
\hline 20 & 426 & $\mathrm{PERDATE}$ & $s t r 1 n g\}$ & $10^{\circ}$ & $\gamma+*$ & DATE $* * *$ \\
\hline
\end{tabular}




\subsection{INSTRUM}

INSTRUM: 11 fields, record length $=383$

\begin{tabular}{|c|c|c|c|c|c|c|}
\hline $\begin{array}{l}\text { Fld } \\
\text { 非 }\end{array}$ & $\begin{array}{c}\text { File } \\
\text { Offset }\end{array}$ & $\begin{array}{l}\text { Field } \\
\text { Name }\end{array}$ & Type & $\begin{array}{l}\text { Screen } \\
\text { Format }\end{array}$ & \multicolumn{2}{|c|}{$\begin{array}{l}\text { Related/Choice } \\
\text { File Field作 }\end{array}$} \\
\hline 0 & 1 & $\overline{\text { Deleted }}$ & if "!" & & & \\
\hline 1 & 2 & INSTRID & Mune 1 cs & 4 & & \\
\hline 2 & 6 & MAKERID & Relational & 8 & ORGANISE & 1 \\
\hline 3 & 14 & MAKER & Relational & 50 & ORGANISE & 3 \\
\hline 4 & 64 & SERIAL & String\$ & 50 & & \\
\hline 5 & 114 & COMMDATE & Strugs & 10 & & \\
\hline 6 & 124 & CALINTER & UCase\$. & 10 & & \\
\hline 7 & 134 & HOTEST1 & Strings & 50 & & \\
\hline 8 & 184 & Hotes2 & String\$ & 50 & & \\
\hline 9 & 234 & HOTES3 & String\$ & 50 & & \\
\hline 10 & 284 & $110 T$ HS 4 . & String\$ & 50 & & \\
\hline 11 & 334 & HOTESS & String\$ & 50 & & \\
\hline
\end{tabular}

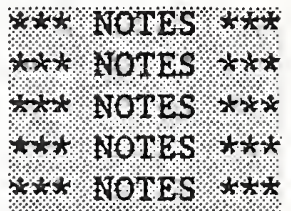

* * IXSTR ID 6 \&

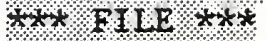

$* \times *$ IEW $\times * x$

**t. OPBRATOR $* * \%$

t.t. VIEW + ttt

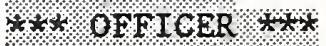

4. 1 YOTES $*$ * $* * *$ NOTES $* * *$ 


\subsection{PRODUCT}

PRODUCT: 19 fields, record length $=494$

\begin{tabular}{|c|c|c|c|c|c|c|c|c|}
\hline Fld & File & Field & & Screen & Related/C & ice & & \\
\hline 非 & Offset & Name & Type & Format & File Fi & & & \\
\hline 0 & 1 & Deleted & if "!" & & & & & \\
\hline 1 & 2 & PRODID & UCase\$ & 78 & & & & \\
\hline 2 & 9 & CHEKPROD & Numerlcs & 8 & & & $x+\pi$ & CHECKSUM $*$ r \\
\hline 3 & 17 & PRODNAME & String\$ & 50 & & & $* x$ & NAME $* * *$ \\
\hline 4 & 67 & MANUFACT & Relational & 40 & ORGANISE & 3 & & \\
\hline 5 & 107 & MANUFID & Relational & 6 & ORGANISE & 1 & & \\
\hline 6 & 113 & CONTACT & Relational & 40 & PEOPLE & 3 & & \\
\hline 7 & 153 & CONTACID & Relational & 6 & PEOPLE & 1 & & \\
\hline 8 & 159 & CATNO & String\$ & 15 & & & & \\
\hline 9 & 174 & MAINMAT & Strings & 50 & & & & \\
\hline 10 & 224 & CONPOS & Logical & 1 & $\mathrm{Y} \mathrm{N}$ & & $x+x$ & COMPOSIT $\times 2 * *$ \\
\hline 11 & 225 & PRODENSI & Single! & 7 & & & $x+x$ & DENSITY + t* \\
\hline 12 & 229 & PROTHICK & Single! & 7 & & & $* x$ & THICK $\times * *$ \\
\hline 13 & 233 & MAIA USE & Choice & 22 & MATIUSE & 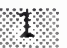 & 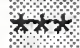 & MAINUSE $-x * *$ \\
\hline 14 & 235 & PRODESSCI & String\$ & 50 & & & 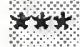 & DESCRIPT $r+\gamma x$ \\
\hline 15 & 285 & PRODESC2 & String\$ & 50 & & & $x * *$ & DESGRIPT $+x+x$ \\
\hline 16 & 335 & PRODESC 3 & Strings & 50 & & & $+x$ & DESCRIPT $* t *$ \\
\hline 17 & 385 & PRODESC4 & String\$ & 50 & & & $x+7$ & DESCRTPT $* x *$ \\
\hline 18 & 435 & PRODESCS & String\$ & 50 & & & $x+x$ & DESCRIPT * *t* \\
\hline 19 & 485 & PRDATE : & Strings & 10 & & & $x+x$ & DATE $x \neq *$ \\
\hline
\end{tabular}

\section{Future Modifications to FDMS Beta Version File Formats}

A review of the file formats in Section 2 indicates the need for additional modifications to the internal storage. Some of these modifications are required in order to provide consistency in the length of relational fields. Other modifications are needed to standardize the naming or type specification of identical fields in separate data files. Finally, some modifications are required to include fields missing from the original design which are necessary for current computer fire models. All modifications are indicated by highlighting the variation of the format in this section from the corresponding specification in the previous section. This section details changes which have not been made at this time but are required in order for the beta version of FDMS to provide acceptable storage of all fire test data during the transition to the next generation of software. Existing import and export software should not be changed until the modifications suggested in this section have been incorporated into the FDMS software.

New file specifications required to include the LIFT (Lateral Ignition and Flame spread Test apparatus) and room/corner test in the FDMS are detailed in this section. Modifications in these formats from the original design specifications are highlighted. 
REMINDER: Highlighting throughout this section indicates future modifications which will be made to the FDMS beta version formats in the previous section.

\subsection{CONE}

CONE: 98 fields, record length $=939$

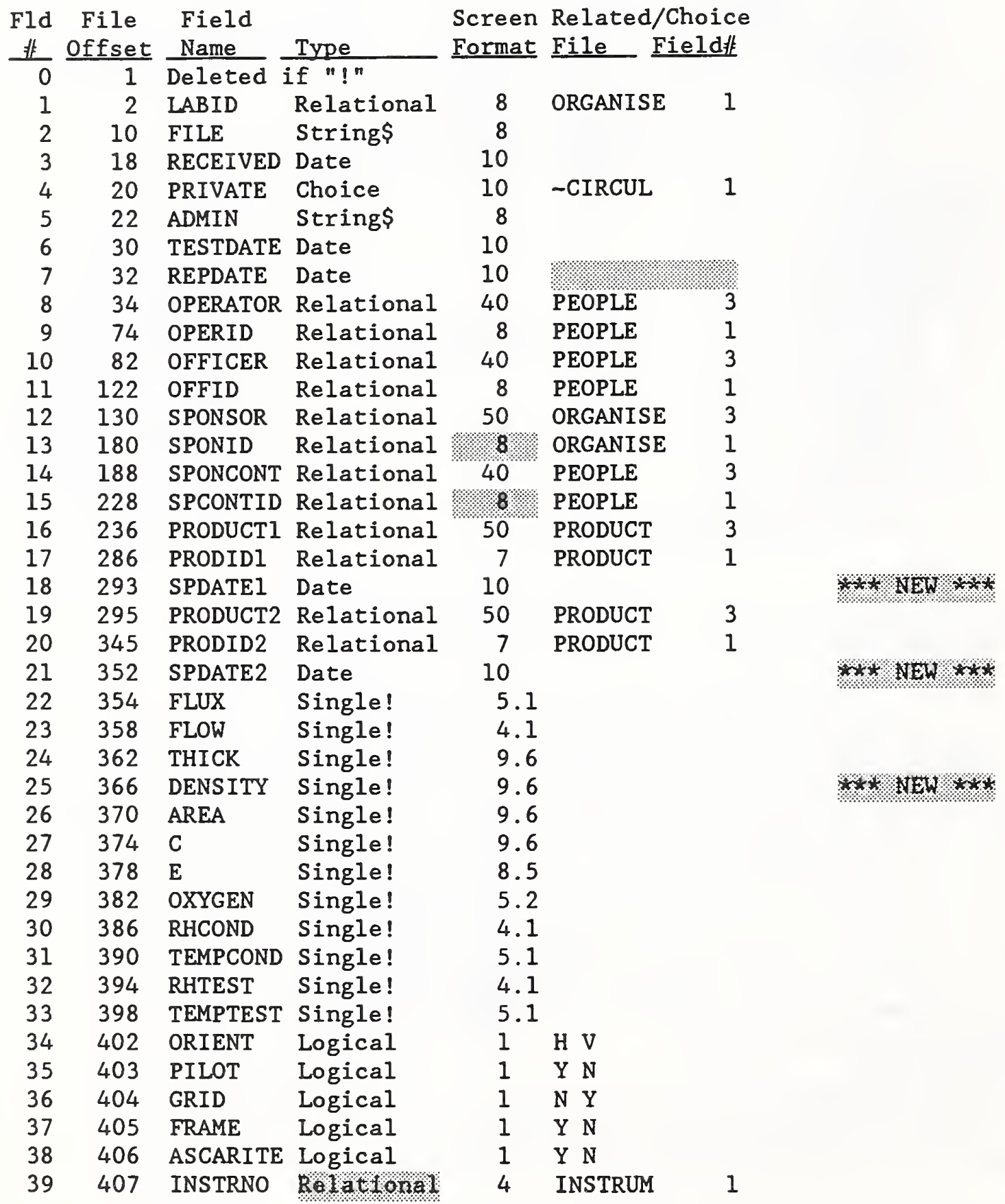




\begin{tabular}{|c|c|c|c|c|c|}
\hline 40 & 411 & SCANS & Integer\% & 4 & \\
\hline 41 & 413 & INTERVAL & Integer\% & 2 & \\
\hline 42 & 415 & COMMENT1 & String\$ & 60 & \\
\hline 43 & 475 & COMMENT2 & Strings & 60 & \\
\hline 44 & 535 & COMMENT3 & String\$ & 60 & \\
\hline 45 & 595 & COMMENT 4 & Strings & 60 & \\
\hline 46 & 655 & COMMENT5 & String\$ & 60 & \\
\hline 47 & 715 & MASSI & Single! & 7.1 & \\
\hline 48 & 719 & MASSF & Single! & 7.1 & \\
\hline 49 & 723 & MASSLOSS & Single! & 7.2 & \\
\hline 50 & 727 & TIGN & Integer\% & 5 & \\
\hline 51 & 729 & FLAMEOUT & Integer\% & 5 & \\
\hline 52 & 731 & MAXTIME & Integer\% & 5 & \\
\hline 53 & 733 & MAXQDOT & Single! & 9.1 & \\
\hline 54 & 737 & MAXMDOT & Single! & 9.2 & \\
\hline 55 & 741 & MAXSIGMA & Single! & 9.2 & \\
\hline 56 & 745 & TOTLHEAT & Single! & 9.2 & \\
\hline 57 & 749 & AVGQDOT & Single! & 9.2 & \\
\hline 58 & 753 & AVGMDOT & Single! & 9.2 & \\
\hline 59 & 757 & AVGHC & Single! & 9.2 & \\
\hline 60 & 761 & AVGSIGLA & Single! & 9.2 & Axt AVSIGM \\
\hline 61 & 765 & AVGCO2 & Single! & 9.5 & \\
\hline 62 & 769 & AVGCO & Single! & 9.5 & \\
\hline 63 & 773 & AVGH2O & Single! & 9.5 & \\
\hline 64 & 777 & QDOT60 & Single! & 9.2 & \\
\hline 65 & 781 & MDOT60 & Single! & 9.2 & \\
\hline 66 & 785 & $\mathrm{HC} 60$ & Single! & 9.2 & \\
\hline 67 & 789 & SIGMA60 & Single! & 9.2 & \\
\hline 68 & 793 & $\mathrm{CO} 260$ & Single! & 9.5 & \\
\hline 69 & 797 & $\mathrm{CO} 60$ & Single! & 9.5 & \\
\hline 70 & 801 & H2O60 & Single! & 9.5 & \\
\hline 71 & 805 & QDOT180 & Single! & 9.2 & \\
\hline 72 & 809 & MDOT180 & Single! & 9.2 & \\
\hline 73 & 813 & HC180 & Single! & 9.2 & \\
\hline 74 & 817 & SIGMA180 & Single! & 9.2 & \\
\hline 75 & 821 & $\mathrm{CO} 2180$ & Single! & 9.5 & \\
\hline 76 & 825 & $\mathrm{CO180}$ & Single! & 9.5 & \\
\hline 77 & 829 & H20180 & Single! & 9.5 & \\
\hline 78 & 833 & QDOT300 & Single! & 9.2 & \\
\hline 79 & 837 & MDOT300 & Single! & 9.2 & \\
\hline 80 & 841 & HC300 & Single! & 9.2 & \\
\hline 81 & 845 & SIGMA 300 & Single! & 9.2 & \\
\hline 82 & 849 & $\mathrm{CO} 2300$ & Single! & 9.5 & \\
\hline 83 & 853 & $\mathrm{CO} 300$ & Single! & 9.5 & \\
\hline 84 & 857 & H2O30O & Single! & 9.5 & \\
\hline 85 & 861 & SOOT & Single! & 9.5 & \\
\hline 86 & 865 & HCL & Single! & 9.5 & \\
\hline 87 & 869 & HCN & Single! & 9.5 & \\
\hline 88 & 873 & HBR & Single! & 9.5 & \\
\hline
\end{tabular}




$\begin{array}{lllll}89 & 877 & \text { TUH } & \text { Single! } & 9.5 \\ 90 & 881 & \text { USER1\$ } & \text { String\$ } & 10 \\ 91 & 891 & \text { USER2\$. } & \text { String\$ } & 10 \\ 92 & 901 & \text { USER3\$ } & \text { String\$ } & 10 \\ 93 & 911 & \text { USERNUM1 } & \text { Single! } & 10.2 \\ 94 & 915 & \text { USERNUM2 } & \text { Single! } & 10.2 \\ 95 & 919 & \text { USERNUM3 } & \text { Single! } & 10.3 \\ 96 & 923 & \text { VERSION } & \text { Numeric\$ } & 5 \\ 97 & 928 & \text { TEST } & \text { Numeric\$ } & 5 \\ 98 & 933 & \text { ZNUMBER } & \text { Numeric\$ } & 7\end{array}$

\subsection{FURN}

The HFF field originally allocated to hydrogen fluoride measurements was reallocated to total unburned hydrocarbons, TUH, to conform to current usage in laboratories.

FURN: 95 fields, record length $=937$

\begin{tabular}{|c|c|c|c|c|c|c|}
\hline$\underset{\text { 非 }}{\mathrm{Fld}}$ & $\begin{array}{l}\text { File } \\
\text { offset }\end{array}$ & $\begin{array}{l}\text { Field } \\
\text { Name }\end{array}$ & Type & $\begin{array}{l}\text { Screen } \\
\text { Format }\end{array}$ & \multicolumn{2}{|c|}{$\begin{array}{l}\text { Related/Choice } \\
\text { File Field非 }\end{array}$} \\
\hline 0 & 1 & Deleted & if "!" & & & \\
\hline 1 & 2 & LABID & Relational & 8 & ORGANISE & 1 \\
\hline 2 & 10 & FILE & Strings & 8 & & \\
\hline 3 & 18 & RECEIVED & Date & 10 & & \\
\hline 4 & 20 & PRIVATE & Choice & 10 & -CIRCUL & 1 \\
\hline 5 & 22 & ADMIN & Strings & 8 & & \\
\hline 6 & 30 & TESTDATE & Dare & 10 & & \\
\hline 7 & 32 & REPDATE & Datec & 10 & & \\
\hline 8 & 34 & OPERATOR & Relational & 40 & PEOPLE & 3 \\
\hline 9 & 74 & OPERID & Relational & 8 & PEOPLE & 1 \\
\hline 10 & 82 & OFFICER & Relational & 40 & PEOPLE & 3 \\
\hline 11 & 122 & OFFID & Relational & 8 & PEOPLE & 1 \\
\hline 12 & 130 & SPONSOR & Relational & 50 & ORGANISE & 3 \\
\hline 13 & 180 & SPONID & Relational & 8 & ORGANISE & 1 \\
\hline 14 & 188 & SPONCONT & Relational & 40 & PEOPLE & 3 \\
\hline 15 & 228 & SPCONTID & Relational & 8 & PEOPLE & 1 \\
\hline 16 & 236 & PRODUCT1 & Relational & 50 & PRODUCT & 3 \\
\hline 17 & 286 & PRODID1 & Relational & 7 & PRODUCT & 1 \\
\hline 18 & 293 & SPDATE1 & Date & 10 & & \\
\hline 19 & 295 & PRODUCT2 & Relational & 50 & PRODUCT & 3 \\
\hline 20 & 345 & PRODID2 & Relational & 7 & PRODUCT & 1 \\
\hline 21 & 352 & SPDATE2 & Date & 10 & & \\
\hline 22 & 354 & FLUX & Single! & 5.1 & & \\
\hline 23 & 358 & FLOW & Single! & 4.1 & & \\
\hline 24 & 362 & THICK & Single! & 9.6 & & \\
\hline 25 & 366 & DENSITY & Single! & 9.6 & & \\
\hline 26 & 370 & AREA & Single! & 9.6 & & \\
\hline 27 & 374 & C & Single! & 9.6 & & \\
\hline
\end{tabular}

How

WHW

$4+1+4+2$ 
Future Modifications to FDMS Beta Version File Formats

\begin{tabular}{|c|c|c|c|c|c|}
\hline 28 & 378 & $E$ & Single! & 8.5 & \\
\hline 29 & 382 & OXYGEN & Single! & 5.2 & \\
\hline 30 & 386 & RHCOND & Single! & 4.1 & \\
\hline 31 & 390 & TEMPCOND & Single! & 5.1 & \\
\hline 32 & 394 & RHTEST & Single! & 4.1 & \\
\hline 33 & 398 & TEMPTEST & Single! & 5.1 & \\
\hline 34 & 402 & IGNTYPE & Choice & 30 & -IGNITOR \\
\hline 35 & 404 & ASCARITE & Logical & 1 & Y N \\
\hline 36 & 405 & INSTRNO & Relat Lonal & 4 & INSTRUM \\
\hline 37 & 409 & SCANS & Integer\% & 4 & \\
\hline 38 & 411 & INTERVAL & Integer\% & 2 & \\
\hline 39 & 413 & COMMENT1 & String\$ & 60 & \\
\hline 40 & 473 & COMMENT 2 & String\$ & 60 & \\
\hline 41 & 533 & COMMENT3 & Strings & 60 & \\
\hline 42 & 593 & COMMENT 4 & String\$ & 60 & \\
\hline 43 & 653 & COMMENTS & String\$ & 60 & \\
\hline 44 & 713 & MASSI & Single! & $1 / 1$ & \\
\hline 45 & 717 & MASSF & Single! & 7.1 & \\
\hline 46 & 721 & MASSLOSS & Single! & 7.2 & \\
\hline 47 & 725 & TIGN & Integer\% & 5 & \\
\hline 48 & 727 & FLAMEOUT & Integer\% & 5 & \\
\hline 49 & 729 & MAXTIME & Integer\% & 5 & \\
\hline 50 & 731 & MAXQDOT & Single! & 9.1 & \\
\hline 51 & 735 & MAXMDOT & Single! & 9.2 & \\
\hline 52 & 739 & MAXS IGMA & Single! & 9.2 & \\
\hline 53 & 743 & TOTLHEAT & Single! & 9.2 & \\
\hline 54 & 747 & AVGQDOT & Single! & 9.2 & \\
\hline 55 & 751 & AVGMDOT & Single! & 9.2 & \\
\hline 56 & 755 & AVGHC & Single! & 9.2 & \\
\hline 57 & 759 & AVGSI CMA & Single! & 9.2 & \\
\hline 58 & 763 & $\mathrm{AVGCO} 2$ & Single! & 9.5 & \\
\hline 59 & 767 & AVGCO & Single! & 9.5 & \\
\hline 60 & 771 & AVGH2O & Single! & 9.5 & \\
\hline 61 & 775 & QD0T60 & Single! & 9.2 & \\
\hline 62 & 779 & MDOT60 & Single! & 9.2 & \\
\hline 63 & 783 & HC60 & Single! & 9.2 & \\
\hline 64 & 787 & SIGMA60 & Single! & 9.2 & \\
\hline 65 & 791 & $\mathrm{CO} 260$ & Single! & 9.5 & \\
\hline 66 & 795 & Co60 & Single! & 9.5 & \\
\hline 67 & 799 & H2O60 & Single! & 9.5 & \\
\hline 68 & 803 & QDOT180 & Single! & 9.2 & \\
\hline 69 & 807 & MDOT180 & Single! & 9.2 & \\
\hline 70 & 811 & $\mathrm{HCl} 80$ & Single! & 9.2 & \\
\hline 71 & 815 & SIGMA180 & Single! & 9.2 & \\
\hline 72 & 819 & $\mathrm{CO} 2180$ & Single! & 9.5 & \\
\hline 73 & 823 & $\mathrm{Co180}$ & Single! & 9.5 & \\
\hline 74 & 827 & H20180 & Single! & 9.5 & \\
\hline 75 & 831 & QDOT300 & Single! & 9.2 & \\
\hline 76 & 835 & MDOT 300 & Single! & 9.2 & \\
\hline
\end{tabular}




\begin{tabular}{|c|c|c|c|c|}
\hline 77 & 839 & НС 300 & Single! & 9.2 \\
\hline 78 & 843 & S IGMA300 & Single! & 9.2 \\
\hline 79 & 847 & $\mathrm{CO} 2300$ & Single! & 9.5 \\
\hline 80 & 851 & $\mathrm{CO} 300$ & Single! & 9.5 \\
\hline 81 & 855 & $\mathrm{H} 20300$ & Single! & 9.5 \\
\hline 82 & 859 & SOOT & Single! & 9.5 \\
\hline 83 & 863 & HCL & Single! & 9.5 \\
\hline 84 & 867 & $\mathrm{HCN}$ & Single! & 9.5 \\
\hline 85 & 871 & HBR & Single! & 9.5 \\
\hline 86 & 875 & KUH & Single! & 9.5 \\
\hline 87 & 879 & USERIS & String\$ & 10 \\
\hline 88 & 889 & Y SER $2 \$$ & String\$ & 10 \\
\hline 89 & 899 & USER3Ş & String\$ & 10 \\
\hline 90 & 909 & USERNUMI & Single! & 10.2 \\
\hline 91 & 913 & USERNUM2 & Single! & 10.2 \\
\hline 92 & 917 & USERNUM3 & Single! & 10.3 \\
\hline 93 & 921 & VERSION & Numeric\$ & 5 \\
\hline 94 & 926 & TEST & Numeric\$ & 5 \\
\hline 95 & 931 & ZNUMBER & Numeric\$ & 7 \\
\hline
\end{tabular}

H.

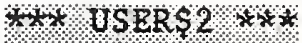

\subsection{LIFT}

The PRODORG1, CONTACT, PRODORG2, and CONTACT2 fields in the original LIFT data file have been deleted since they are included in the PRODUCT file as the MUNTFID and CONTACID fields.

LIFT: 57 fields, record length $=796$

\begin{tabular}{|c|c|c|c|c|c|c|}
\hline Fld & File & Field & & Screen & \multicolumn{2}{|c|}{ Related/Choice } \\
\hline 非 & Offset & Name & Type & Format & File & d非 \\
\hline 0 & 1 & Deleted & if $"$ ! & & & \\
\hline 1 & 2 & LABID & Relational & 8 & ORGANISE & 1 \\
\hline 2 & 10 & FILE & string\$ & 8 & & \\
\hline 3 & 18 & RECEIVED & Date & 10 & & \\
\hline 4 & 20 & PRIVATE & Choice & 10 & - CIRUMU & $1 \%$ \\
\hline 5 & 22 & ADMIN & String\$ & 8 & & \\
\hline 6 & 30 & TESTDATE & Date & 10 & & \\
\hline 7 & 32 & REPDATE & Date & 10 & & \\
\hline 8 & 34 & OPERATOR & Relational & 40 & PEOPLE & 3 \\
\hline 9 & 74 & $O \mathrm{EROD}=$ & Relational & 8 & PEOPLE & 1 \\
\hline 10 & 82 & OFFICER & Relational & 40 & PEOPLE & 3 \\
\hline 11 & 122 & $0 H_{1}$ & Relational & 8 & PEOPLE & 1 \\
\hline 12 & 130 & SPONSOR & Relational & 50 & ORGANISE & 3 \\
\hline 13 & 180 & SPONID & Relational & 8 & ORGANISE & 1 \\
\hline 14 & 188 & SPONCONT & Relational & 40 & PEOPLE & 3 \\
\hline 15 & 228 & SPCONIID & Relational & 8 & PEOPLE & 1 \\
\hline 16 & 236 & PRODUCTI & Relational & 50 & PRODUCT & 3 \\
\hline 17 & 286 & PRODIDI & Relational & 7 & PRODUCT & 1 \\
\hline
\end{tabular}

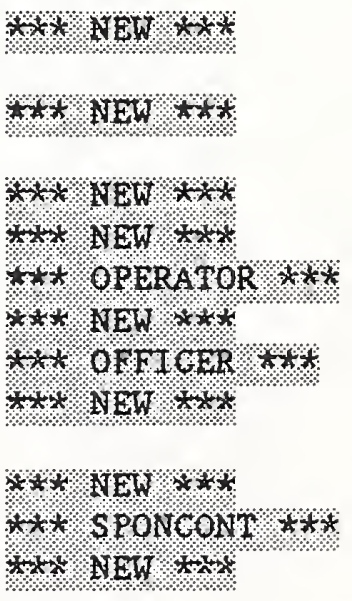


Future Modifications to FDMS Beta Version File Formats

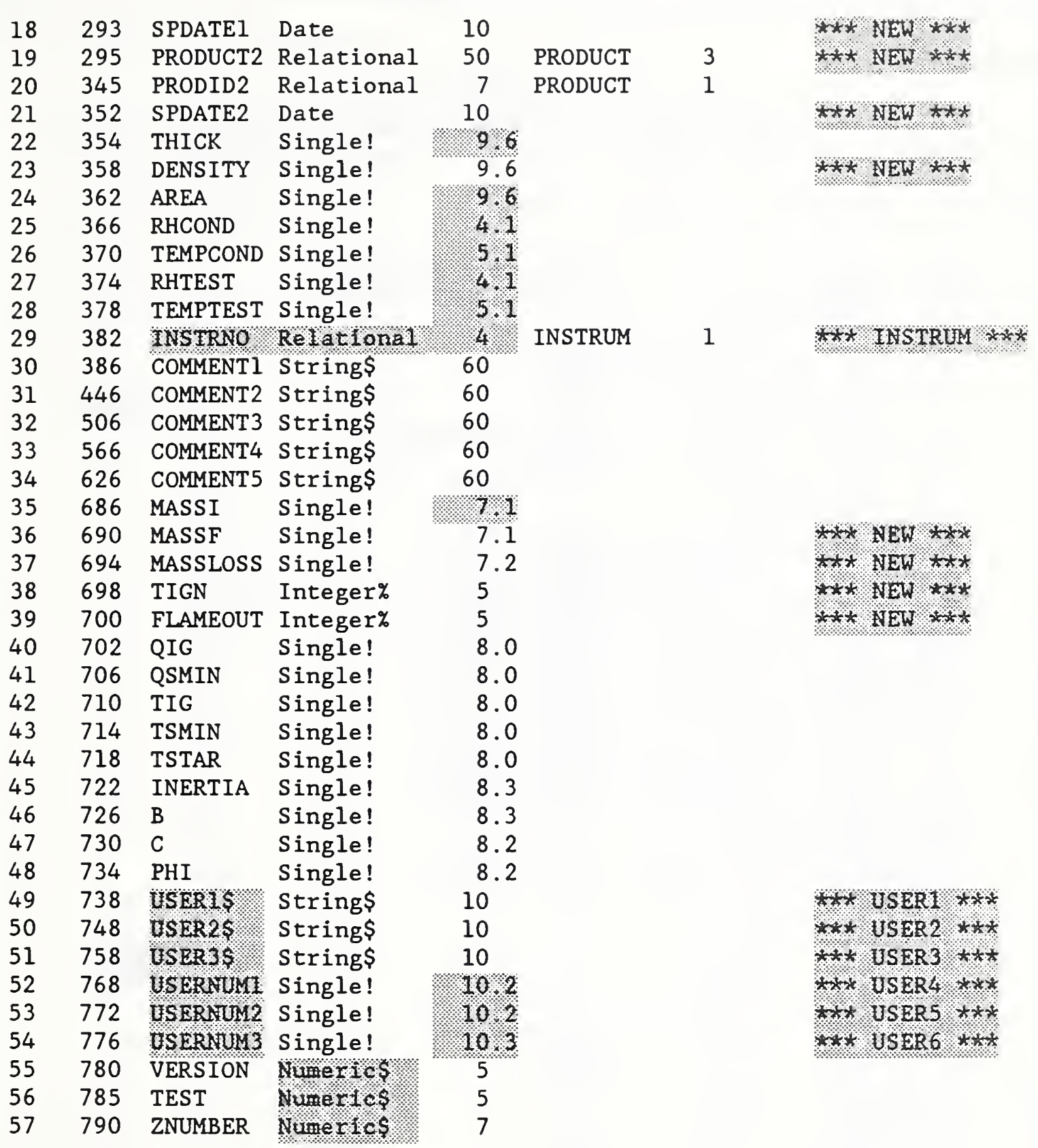




\subsection{ROOM}

The HIF field originally allocated to hydrogen fluoride measurements was reallocated to total unburned hydrocarbons, WUI, to conform to current usage in laboratories.

ROOM: 102 fields, record length $=1129$

\begin{tabular}{|c|c|c|c|c|c|c|}
\hline $\begin{array}{l}\text { F1d } \\
\text { 非 }\end{array}$ & $\begin{array}{c}\text { File } \\
\text { Offset }\end{array}$ & $\begin{array}{l}\text { Field } \\
\text { Name } \\
\end{array}$ & Type & $\begin{array}{l}\text { Screen } \\
\text { Format }\end{array}$ & $\begin{array}{l}\text { Related/ } \\
\text { File E }\end{array}$ & \\
\hline 0 & 1 & Deleted & if $"$ !" & & & \\
\hline 1 & 2 & LABID & Relational & $8:$ & ORGANISE & 1 \\
\hline 2 & 10 & FILE & String\$ & 8. & & \\
\hline 3 & 18 & RECEIVED & Date & 10 & & \\
\hline 4 & 20 & PRIVATE & Choice & 10 & G. W oul & 1 \\
\hline 5 & 22 & ADMIN & String\$ & 8. & & \\
\hline 6 & 30 & TESTDATE & Date & 10 & & \\
\hline 7 & 32 & REPDATE & Date & 10 & & \\
\hline 8 & 34 & OPERATOR & Relational & 40 & PEOPLE & 3 \\
\hline 9 & 74 & OPERID & Relational & 8 & PEOPLE & 1 \\
\hline 10 & 82 & OFFICER & Relational & 40 & PEOPLE & 3 \\
\hline 11 & 122 & OFFID & Relational & 8.8 & PEOPLE & 1 \\
\hline 12 & 130 & SPONSOR & Relational & 50 & ORGANISE & 3 \\
\hline 13 & 180 & SPONID & Relational & 8 & ORGANISE & 1 \\
\hline 14 & 188 & SPONCONT & Relational & 48 & PEOPLE & 3 \\
\hline 15 & 228 & SPCONTID & Relational & 8 & PEOPLE & 1 \\
\hline 16 & 236 & PRODUCT1 & Relational & 50. & PRODUCT & 3 \\
\hline 17 & 286 & PRODID1 & Relational & 7 & PRODUCT & 1 \\
\hline 18 & 293 & SPDATE1 & Date & 10 & & \\
\hline 19 & 295 & PRODUCT2 & Relational & 80. & PRODUCT & 3 \\
\hline 20 & 345 & PRODID2 & Relational & 7 & PRODUCT & 1 \\
\hline 21 & 352 & SPDATE2 & Date & 10 & & \\
\hline 22 & 354 & FLUX & Single! & 5.1 & & \\
\hline 23 & 358 & FLOW & Single! & 4.1 & & \\
\hline 24 & 362 & THICK & Single! & 9.6 & & \\
\hline 25 & 366 & DENSITY & Single! & 9.6 & & \\
\hline 26 & 370 & AREA & Single! & 6.6 & & \\
\hline 27 & 374 & E & Single! & 8. 8 & & \\
\hline 28 & 378 & OXYGEN & Single! & 5.2 & & \\
\hline 29 & 382 & RHCOND & Single! & 7. 1 & & \\
\hline 30 & 386 & TEMPCOND & Single! & 5. 1 & & \\
\hline 31 & 390 & RHTEST & Single! & 4. 1 & & \\
\hline 32 & 394 & TEMPTEST & Single! & 5.1 & & \\
\hline 33 & 398 & SURFDENS & Single! & 9.6 & & \\
\hline 34 & 402 & MOUNT1 & String\$ & 60 & & \\
\hline 35 & 462 & MOUNT2 & String\$ & 60 & & \\
\hline 36 & 522 & IGNITOR & Choice & 1 & $S A O$ & 1 \\
\hline 37 & 524 & BURNER & String\$ & 60 & & \\
\hline 38 & 584 & LOCATION & Choice & 1 & C W 0 & 1 \\
\hline
\end{tabular}


Future Modifications to FDMS Beta Version File Formats

\begin{tabular}{|c|c|c|c|c|c|c|c|c|c|}
\hline 39 & 586 & ASCARITE & Logical & 1 & Y N & & & & \\
\hline 40 & 587 & INSTRNO & Retational & 4 & IHSTRIAY & 1 & $x * x$ & INST & CRUM $* * *$ \\
\hline 41 & 591 & SCANS & Integer\% & 4 & & & & & \\
\hline 42 & 593 & INTERVAL & Integer\% & 2 & & & & & \\
\hline 43 & 595 & COMMENT1 & String\$ & 60 & & & & & \\
\hline 44 & 655 & COMMENT2 & Strings & 60 & & & & & \\
\hline 45 & 715 & COMMENT 3 & String\$ & 60 & & & & & \\
\hline 46 & 775 & COMMENT4 & String\$ & 60 & & & & & \\
\hline 47 & 835 & COMMENTS & String\$ & 60 & & & & & \\
\hline 48 & 895 & MASSI & Single! & 1.1 & & & & & \\
\hline 49 & 899 & MASSF & Single! & 7.1 & & & & & \\
\hline 50 & 903 & MASSLOSS & Single! & 7.2 & & & $x+1$ & NEW: & $* x$ \\
\hline 51 & 907 & TIGN & Integer\% & 5 & & & & & \\
\hline 52 & 909 & FLASH & Integer\% & 5 & & & & & \\
\hline 53 & 911 & FLAMEOUT & Integer\% & 5 & & & & & \\
\hline 54 & 913 & MAXTIME & Integer\% & 5 & & & & & \\
\hline 55 & 915 & MAXQDOT & Single! & 91 & & & & & \\
\hline 56 & 919 & MAXMDOT & Single! & 9.2 & & & & & \\
\hline 57 & 923 & MAXSIGMA & Single! & 9.2 & & & $*+x$ & NEW: & $+x-x$ \\
\hline 58 & 927 & MAXEXT & Single! & 9.2 & & & & & \\
\hline 59 & 931 & TOTUHEAT & Single! & 9,2 & & & $x+1$ & SUMO & $4 * x$ \\
\hline 60 & 935 & SUMEXT & Single! & 9.2 & & & & & \\
\hline 61 & 939 & AVGQDOT & Single! & 9.2 & & & $* * *$ & NEW & $* x+x$ \\
\hline 62 & 943 & AVGMDOT & Single! & 9.2 & & & $t+t$ & NEH & $t+7$ \\
\hline 63 & 947 & AVGHC & Single! & 92 & & & & & \\
\hline 64 & 951 & AVGS IGMA & Single! & 9,2 & & & & & \\
\hline 65 & 955 & AVGCO2 & Single! & 9.5 & & & & & \\
\hline 66 & 959 & AVGCO & Single! & 9.5 & & & & & \\
\hline 67 & 963 & AVGH 20 & Single! & 9,5 & & & & & \\
\hline 68 & 967 & QDOT60 & Single! & 9.2 & & & $* * *$ & XIEW & $* * *$ \\
\hline 69 & 971 & MDOT60 & Single! & 9.2 & & & $* x+x$ & AEW & $x+x$ \\
\hline 70 & 975 & HC60 & Single! & 9.2 & & & $t+t$ & VEET? & $+*$ \\
\hline 71 & 979 & SIGMA60 & Single! & 9.2 & & & $x+t$ & UEW. & $t x+$ \\
\hline 72 & 983 & $\mathrm{CO} 260$ & Single! & 9.5 & & & $* x+$ & NEW. & $x+*$ \\
\hline 73 & 987 & $\mathrm{CO} 60$ & Single! & 9.5 & & & $* x+x$ & NBW & $x+x$ \\
\hline 74 & 991 & H2060 & Single! & 9.5 & & & $t * t$ & NEW & $t+t$ \\
\hline 75 & 995 & QDOT180 & Single! & 9.2 & & & $* x$ & IEW & $t+*$ \\
\hline 76 & 999 & MDOT 180 & Single! & 9.2 & & & $x+x$ & NEW: & $t+t$ \\
\hline 77 & 1003 & HC180 & Single! & 9.2 & & & $* x+2$ & yew & $* x+*$ \\
\hline 78 & 1007 & SIGMA 180 & Single! & 9.2 & & & $6 x+$ & NEW & $t * *$ \\
\hline 79 & 1011 & $\mathrm{CO} 2180$ & Single! & 9.5 & & & $x+x$ & NEW & $x+*$ \\
\hline 80 & 1015 & $\mathrm{C} 0180$ & Single! & 9.5 & & & $x+x$ & NEW & 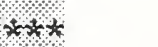 \\
\hline 81 & 1019 & $\mathrm{H} 20180$ & Single! & 9.5 & & & $* * x$ & NEW & $x+x$ \\
\hline 82 & 1023 & QDOT 300 & Single! & 9.2 & & & $x+t$ & NEW & $t+x$ \\
\hline 83 & 1027 & MDOT 300 & Single! & 9.2 & & & $* * *$ & NEW. & $x+*$ \\
\hline 84 & 1031 & $\mathrm{HC} 300$ & Single! & 9.2 & & & $* * *$ & NEW & $* x *$ \\
\hline 85 & 1035 & SIGMA 300 & Single! & 9.2 & & & $x+x$ & NEW & $* x+x$ \\
\hline 86 & 1039 & $\mathrm{CO} 2300$ & Single! & 9.5 & & & $6+x$ & NEW. & $t x t$ \\
\hline 87 & 1043 & $\mathrm{CO} 300$ & Single! & 9.5 & & & $x+x$ & NEW & $x+*$ \\
\hline
\end{tabular}




\begin{tabular}{|c|c|c|c|c|}
\hline 88 & 1047 & $\mathrm{H} 20300$ & Single! & 9.5 \\
\hline 89 & 1051 & SOOT & Single! & 80 \\
\hline 90 & 1055 & HCL & Single! & $9 \%$ \\
\hline 91 & 1059 & $\mathrm{HCN}$ & Single! & 9.5 \\
\hline 92 & 1063 & HBR & Single! & 9.5 \\
\hline 93 & 1067 & IU⿴囗十 & Single! & 9 \\
\hline 94 & 1071 & USERIS & string\$ & 10 \\
\hline 95 & 1081 & LSER2S & String\$ & 10 \\
\hline 96 & 1091 & USER SS & String\$ & 10 \\
\hline 97 & 1101 & USBRAUMI & Single! & $10 \%$ \\
\hline 98 & 1105 & USBRNUK2 & Single! & 10.2 \\
\hline 99 & 1109 & US ERNUKS & Single! & 10 \\
\hline 100 & 1113 & VERSION & Nuiler tcs & 5 \\
\hline 101 & 1118 & TEST & Numerics & 5 \\
\hline 102 & 1123 & ZNUMBER & Huner $1<\%$ & 7 \\
\hline
\end{tabular}

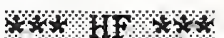

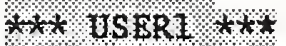

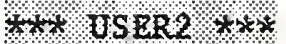

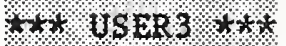

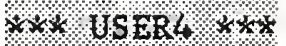

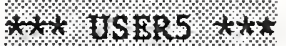

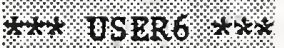

\subsection{FRESIST}

FRESIST: 21 fields, record length $=640$

\begin{tabular}{|c|c|c|c|c|c|c|}
\hline F1d & $\begin{array}{c}\text { File } \\
\text { offset }\end{array}$ & $\begin{array}{l}\text { Field } \\
\text { Name }\end{array}$ & Type & $\begin{array}{l}\text { Screen } \\
\text { Format }\end{array}$ & \multicolumn{2}{|c|}{$\begin{array}{l}\text { Related/Choice } \\
\text { File Field非 }\end{array}$} \\
\hline 0 & 1 & Deleted & if n!" & & & \\
\hline 1 & 2 & TESTCODE & Numeric\$ & 8 & & \\
\hline 2 & 10 & TDATE & Date & 10 & & \\
\hline 3 & 12 & SPONREF & Relational & $8 \%$ & ORGANISE & 1 \\
\hline 4 & 17 & SPONSOR & Relational & 50 & ORGANISE & 3 \\
\hline 5 & 67 & INSUTIME & Integer\% & 3 & & \\
\hline 6 & 69 & INSUCOND & String\$ & 22 & & \\
\hline 7 & 91 & INTETIME & Integer\% & 3 & & \\
\hline 8 & 93 & INTECOND & String\$ & 22 & & \\
\hline 9 & 115 & STABTIME & Integer\% & 3 & & \\
\hline 10 & 117 & STABCOND & String\$ & 22 & & \\
\hline 11 & 139 & TESTTYPE & UCase\$ & 2 & & \\
\hline 12 & 141 & PRODREF & Relational & 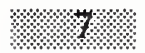 & PRODUCT & 1 \\
\hline 13 & 146 & FRDESC1 & String\$ & 55 & & \\
\hline 14 & 201 & FRDESC2 & String\$ & 55 & & \\
\hline 15 & 256 & FRDESC 3 & Strings & 55 & & \\
\hline 16 & 311 & FRDESC4 & String\$ & 55 & & \\
\hline 17 & 366 & FRDESC5 & String\$ & 55 & & \\
\hline 18 & 421 & FRDESC6 & String\$ & 55 & & \\
\hline 19 & 476 & FRDESC7 & String\$ & 55 & & \\
\hline 20 & 531 & FRDESC 8 & String\$ & 55 & & \\
\hline 21 & 586 & FRDESC 9 & String\$ & 55 & & \\
\hline
\end{tabular}




\subsection{ORGANISE}

\begin{tabular}{|c|c|c|c|c|}
\hline 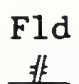 & $\begin{array}{l}\text { File } \\
\text { Offset }\end{array}$ & $\begin{array}{l}\text { Field } \\
\text { Name }\end{array}$ & Type & $\begin{array}{l}\text { Screen Related/Choice } \\
\text { Format File Field非 }\end{array}$ \\
\hline 0 & 1 & $\overline{\text { Deleted }}$ & if $"$ ! " & \\
\hline 1 & 2 & ORGID & Numer 1 cS & 8 \\
\hline 2 & 10 & CHEKORG & Numeric\$ & 8 \\
\hline 3 & 18 & ORGANISE & Strings & 50 \\
\hline 4 & 68 & DIVISION & Strings & 50 \\
\hline 5 & 118 & ADDRESS 1 & String\$ & 32 \\
\hline 6 & 150 & ADDRESS2 & 2 strings & 32 \\
\hline 7 & 182 & CITY & String\$ & 20 \\
\hline 8 & 202 & REGION & String\$ & 12 \\
\hline 9 & 214 & POSTCODE & UCase\$ & 10 \\
\hline 10 & 224 & COUNTRY & Hoases: & 20 \\
\hline 11 & 244 & PHONE & Numeries & 15 \\
\hline 12 & 259 & $\mathrm{FAX}$ & Numeric\$ & 15 \\
\hline 13 & 274 & TELEX & UCase\$ & 15 \\
\hline 14 & 289 & ORGDATE & Date: & 10 \\
\hline
\end{tabular}

\subsection{PEOPLE}

\begin{tabular}{|c|c|c|c|c|}
\hline $\begin{array}{l}\text { F1d } \\
\text { 非 }\end{array}$ & $\begin{array}{l}\text { File } \\
\text { Offset }\end{array}$ & $\begin{array}{l}\text { Field } \\
\text { Name }\end{array}$ & Type & $\begin{array}{l}\text { Screen Related/Choice } \\
\text { Format File Field非 }\end{array}$ \\
\hline 0 & 1 & $\overline{\text { Deleted }}$ & if "!" & \\
\hline 1 & 2 & PERSONID & Nituler $1 \subset s$ & 8 \\
\hline 2 & 10 & CHEKPER & Numeric\$ & 8 \\
\hline 3 & 18 & FULINAME & String\$ & 40 \\
\hline 4 & 58 & FIRSTNAM & String\$ & 12 \\
\hline 5 & 70 & INITIAL & String\$ & 3 \\
\hline 6 & 73 & LASTNAME & String\$ & 20 \\
\hline 7 & 93 & ORGANISE & String\$ & 50 \\
\hline 8 & 143 & DIVISION & String\$ & 50 \\
\hline 9 & 193 & ADDRESS 1 & String\$ & 32 \\
\hline 10 & 225 & ADDRESS2 & string\$ & 32 \\
\hline 11 & 257 & CITY & String\$ & 20 \\
\hline 12 & 277 & REGION & String\$ & 12 \\
\hline 13 & 289 & POSTCODE & UCase & 10 \\
\hline 14 & 299 & COUNTRY & UCases. & 20 \\
\hline 15 & 319 & ADDINFO & strings & 50 \\
\hline 16 & 369 & PHONE & Nume $r$ lcs & 15 \\
\hline 17 & 384 & MORPHONE & Ucase\$: & 18 \\
\hline 18 & 402 & FAX & Humerics & 15 \\
\hline 19 & 417 & TELEX & UCases & 15 \\
\hline 20 & 432 & PERDATE & Date: & 10 \\
\hline
\end{tabular}




\subsection{INSTRUM}

INSTRUM: 11 fields, record length $=375$

\begin{tabular}{|c|c|c|c|c|c|c|}
\hline $\begin{array}{l}\text { F1d } \\
\text { 非 }\end{array}$ & $\begin{array}{c}\text { File } \\
\text { Offset }\end{array}$ & $\begin{array}{l}\text { Field } \\
\text { Name }\end{array}$ & Type & $\begin{array}{l}\text { Screen } \\
\text { Format }\end{array}$ & \multirow{2}{*}{\multicolumn{2}{|c|}{$\begin{array}{l}\text { Related/Choice } \\
\text { File Field非 }\end{array}$}} \\
\hline 0 & 1 & Deleted & if "!" & & & \\
\hline 1 & 2 & INSTRID & Numeric\$ & 4 & & \\
\hline 2 & 6 & MAKERID & Relational & 8 & ORGANISE & 1 \\
\hline 3 & 14 & MAKER & Relational & 50 & ORGANISE & 3 \\
\hline 4 & 64 & SERIAL & String\$ & 50 & & \\
\hline 5 & 114 & COMMDATE & Deter. & 10 & & \\
\hline 6 & 116 & CALINTER & UCase\$ & 10 & & \\
\hline 7 & 126 & NOTES 1 & string\$ & 50 & & \\
\hline 8 & 176 & NOTES 2 & String\$ & 50 & & \\
\hline 9 & 226 & NOTES3 & String\$ & 50 & & \\
\hline 10 & 276 & NOTES4 & String\$ & 50 & & \\
\hline 11 & 326 & NOTES5 & String\$ & 50 & & \\
\hline
\end{tabular}

\subsection{CALIB}

CALIB: 17 fields, record length $=305$

\begin{tabular}{|c|c|c|c|c|c|c|}
\hline $\begin{array}{l}\text { F1d } \\
\text { 非 }\end{array}$ & $\begin{array}{c}\text { File } \\
\text { Offset }\end{array}$ & $\begin{array}{l}\text { Field } \\
\text { Name }\end{array}$ & Type & $\begin{array}{l}\text { Screen } \\
\text { Format }\end{array}$ & \multirow{2}{*}{\multicolumn{2}{|c|}{$\begin{array}{l}\text { Related/Choice } \\
\text { File Field非 }\end{array}$}} \\
\hline 0 & 1 & $\overline{\text { Deleted }}$ & if "!" & & & \\
\hline 1 & 2 & CALIBREF & Relational & 举 & INSTRUM & M \\
\hline 2 & 6 & CALFILE & String\$ & 20 & & \\
\hline 3 & 26 & CALDATE & Deter $、$ : & 10 & & \\
\hline 4 & 28 & NEXTDATE & Datee & 10 & & \\
\hline 5 & 30 & OPERATOR & Relational & 40 & PEOPLE & 3 \\
\hline 6 & 70 & OPERID & Relational & x & PEOPLE & 1 \\
\hline 7 & 78 & OFFICER & Relational & 40 & PEOPLE & 3 \\
\hline 8 & 118 & OFFID & Relational & 6. & PEOPLE & 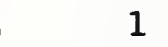 \\
\hline 9 & 126 & CONV & String\$ & 6 & & \\
\hline 10 & 132 & CONSTO & Numeric\$ & 9 & & \\
\hline 11 & 141 & CONST1 & Numeric\$ & 9 & & \\
\hline 12 & 150 & CONST2 & Numeric\$ & 9 & & \\
\hline 13 & 159 & CONST3 & Numeric\$ & 9 & & \\
\hline 14 & 168 & CONST4 & Mune 1 . 5 & 9 & & \\
\hline 15 & 177 & CONST5 & Numerics & 9 & & \\
\hline 16 & 186 & CALNOTE1 & String\$ & 60 & & \\
\hline 17 & 246 & CALNOTE2 & String\$ & 60 & & \\
\hline
\end{tabular}




\subsection{PRODUCT}

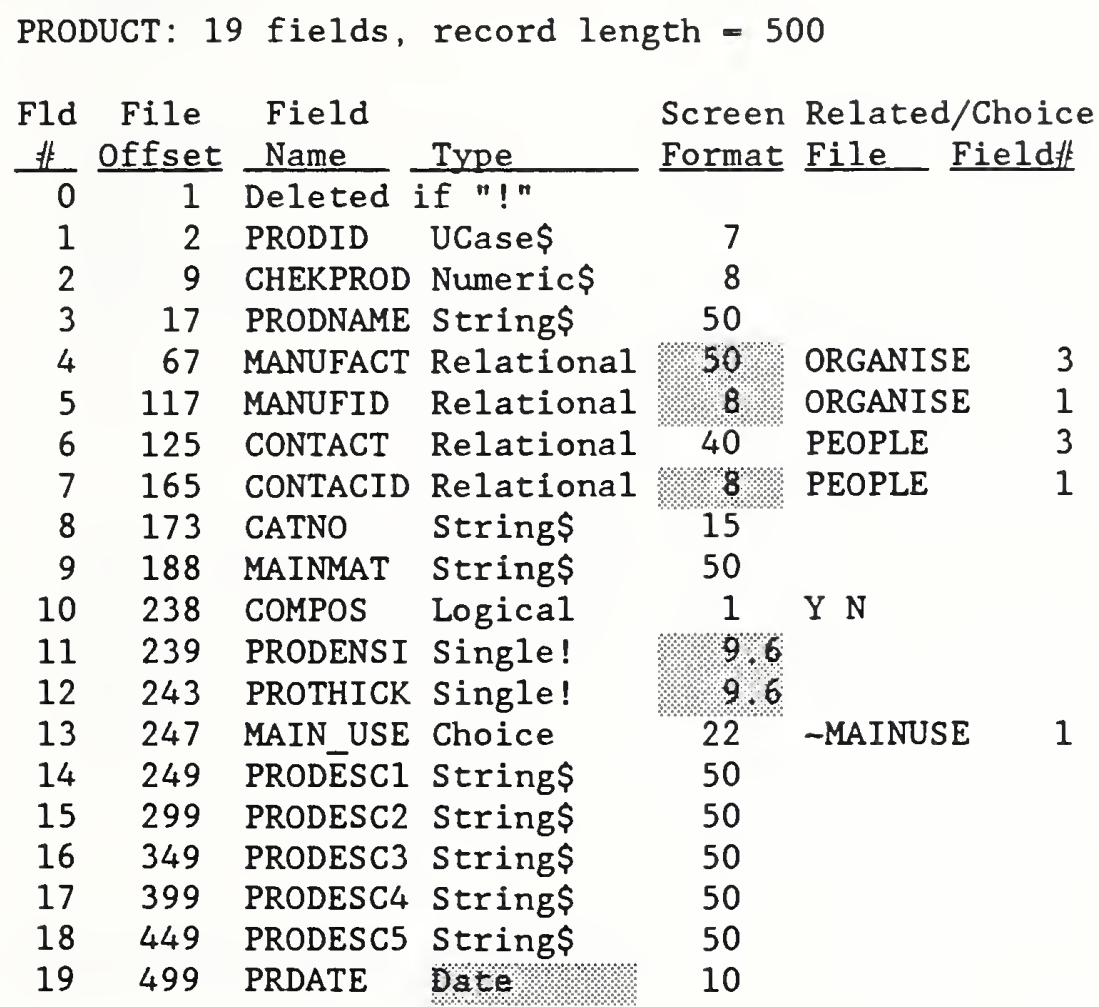

\section{FDMS Beta Version Raw Data File}

The example raw data file from the original FDMS specification document is included in this section highlighting modifications required for use in the FDMS beta version program. This section is included for developers responsible for generating files in the FDMS format. Modifications to the FDMS database from Section 2 have been included. These changes involve the field headings for the scalar data which must be identical to the corresponding names in the database.

RAWCONE
TABIEE
CONE
FILE
34A-FG
SFOIID
U1234567
STCOATID
U2345678
LABID
U3456789
TESTDATE




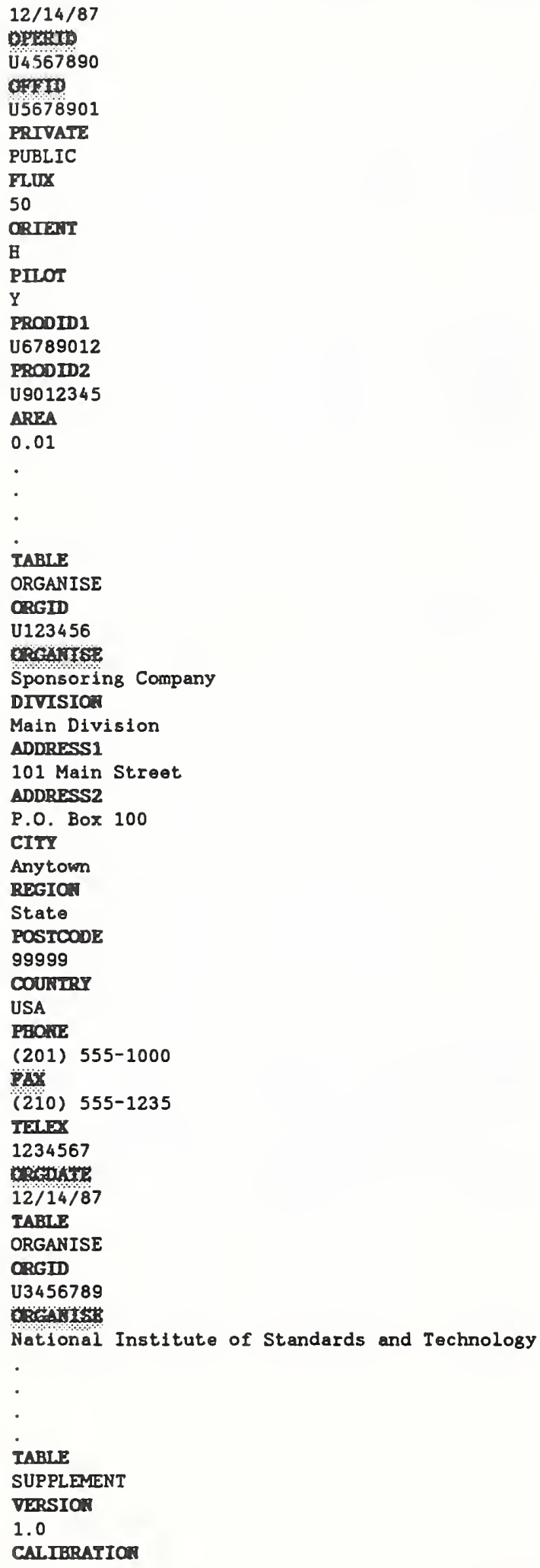




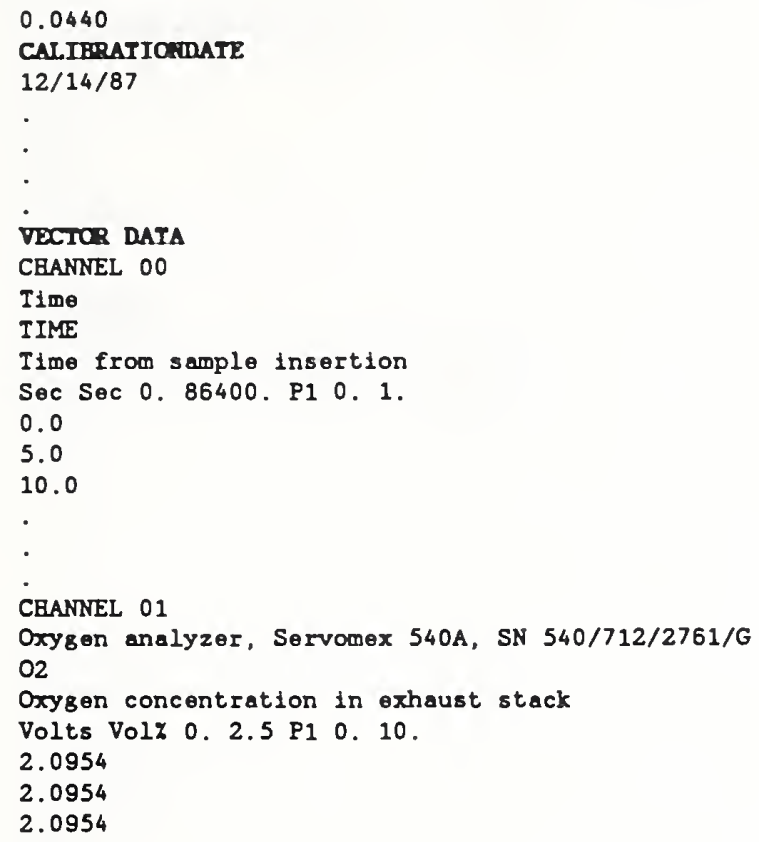

\section{FDMS Beta Version Import/Export File}

The example FDMS import/export file from the original FDMS specification document is included in this section with required modifications highlighted. This section is included for developers responsible for generating files in the FDMS format. Modifications to the FDMS database from Section 2 have been included. These changes involve the field headings for the scalar data which must be identical to the corresponding names in the database.

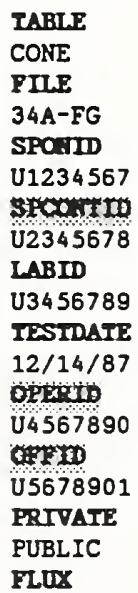




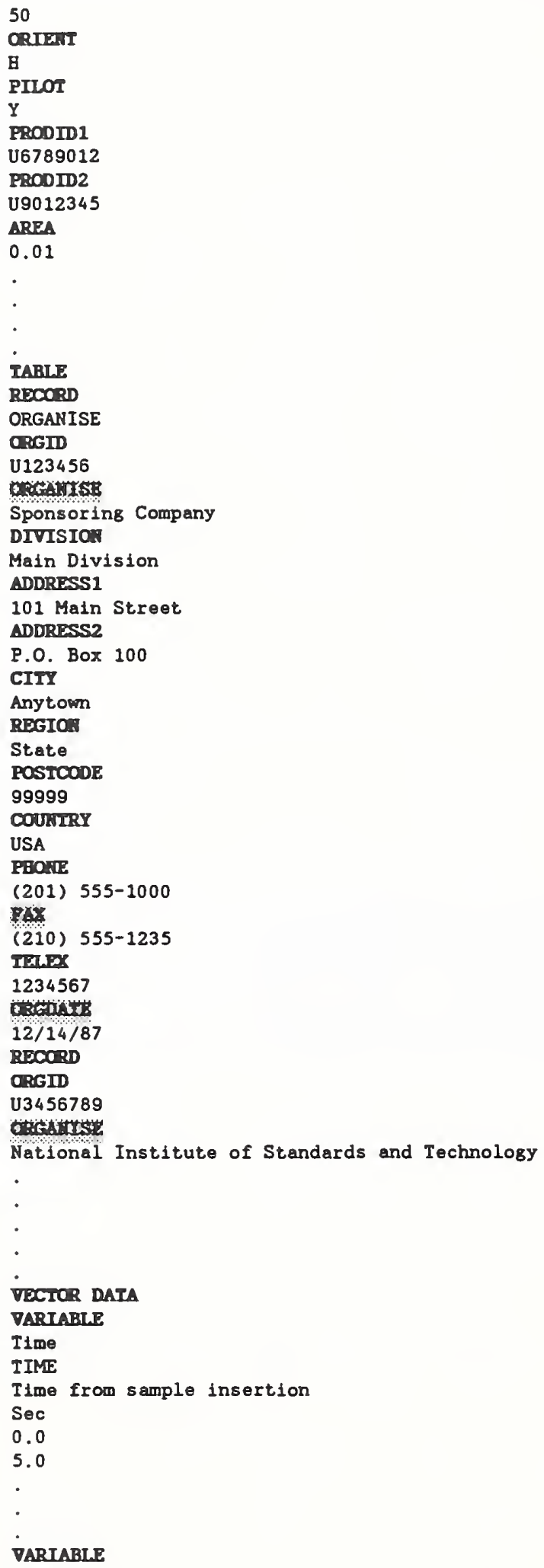

0.0

5.0

vartable 
Load Co11, ATC 6005C06E1XX, SN 2851

MASS

Spectmen mass

Grams

169.85

169.50

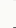

VARIABLE.

Smoke extinction laser system, SSDCLOI

EXT. COEFF .

Smoke extinction coefficient in exhaust stack

$1 / m$

0.0000

0.0000

.

.

VARIABLE

DERIVED

CO2 YIELD

Carbon dioxide yield

$\mathbf{k g} / \mathrm{kg}_{\mathrm{B}}$

0.0000

0.0000 


\section{Field Definitions}

\begin{tabular}{|c|c|c|}
\hline Field Name & Table & Description \\
\hline ADDINFO & PEOPLE & Supplementary information about an individual. \\
\hline ADDRESS1 & ORGANISE, PEOPLE & Street address. \\
\hline ADDRESS2 & ORGANISE, PEOPLE & Additional mailing information \\
\hline ADMIN & CONE, FURN, LIFT, ROOM & $\begin{array}{l}\text { Laboratory specific code used to store internal administrative } \\
\text { information such as Cost Center code or invoice number. }\end{array}$ \\
\hline AREA & CONE, FURN, LIFT, ROOM & $\begin{array}{l}\text { Specimen area }\left(\mathrm{m}^{2}\right) \text {. For the Cone Calorimeter, the area under } \\
\text { the specimen holder edge or the edge frame is not included. }\end{array}$ \\
\hline ASCARITE & CONE, FURN, LIFT, ROOM & $\begin{array}{l}\text { Indicates if the } \mathrm{CO}_{2} \text { was removed from the sample before } \mathrm{O}_{2} \\
\text { was measured using Ascarite or equivalent means. }\end{array}$ \\
\hline AVGCO & CONE, FURN, ROOM & Test average of the $\mathrm{CO}$ yield $(\mathrm{kg} / \mathrm{kg})$. \\
\hline AVGCO2 & CONE, FURN, ROOM & Test average of the $\mathrm{CO}_{2}$ yield $(\mathrm{kg} / \mathrm{kg})$. \\
\hline AVGH2O & CONE, FURN, ROOM & Test average of the $\mathrm{H}_{2} \mathrm{O}$ yield $(\mathrm{kg} / \mathrm{kg})$. \\
\hline AVGHC & CONE, FURN, ROOM & Test average of the effective heat of combustion $\Delta \mathrm{h}_{\mathrm{c}}(\mathrm{kJ} / \mathrm{g})$. \\
\hline AVGMDOT & CONE, FURN, ROOM & Test average of the mass loss rate $\mathrm{m}^{\prime \prime}\left(\mathrm{g} / \mathrm{s} \cdot \mathrm{m}_{2}\right)$ \\
\hline AVGQDOT & CONE, FURN, ROOM & Test average of the rate of heat release $q^{n}\left(\mathrm{~kW} / \mathrm{m}^{2}\right)$. \\
\hline AVGSIGMA & CONE, FURN, ROOM & Test average of the specific smoke extinction area $\sigma_{\mathrm{m}}\left(\mathrm{m}^{2} / \mathrm{kg}\right)$. \\
\hline B & LIFT & Ignition parameter $\left(\mathrm{s}^{-0.5}\right)$. \\
\hline BURNER & ROOM & $\begin{array}{l}\text { When the ignitor is a burner, the heat output values used for the } \\
\text { burner program have to be specified. These are entered as a } \\
\text { string of numbers, separated by at least one blank. The order is: } \\
\text { Time (s) Output (kW) Time (s) Output (kW) ... }\end{array}$ \\
\hline C & CONE, FURN, LIFT & $\begin{array}{l}\text { Parameter. For the Cone Calorimeter, this is the orifice } \\
\text { constant as determined from the } \mathrm{CH}_{4} \text { burner calibration. For } \\
\text { the LIFT, this is the slope of correlated flame spread data }\left(\mathrm{s}^{1 / 2} \text {. }\right. \\
\left.\mathrm{m}^{1 / 2} \cdot \mathrm{W}^{-1}\right) \text {. }\end{array}$ \\
\hline CALDATE & CALIB & Date of the last calibration. \\
\hline CALFILE & CALIB & $\begin{array}{l}\text { Reference field indicating where the original or official } \\
\text { calibration report may be found, e.g., a report number or a } \\
\text { notebook page. Most laboratories will have a different system } \\
\text { for doing this. }\end{array}$ \\
\hline CALIBREF & CALIB & INSTRID number from the INSTRUM table. \\
\hline CALINTER & INSTRUM & Recommended calibration interval in months. \\
\hline
\end{tabular}


Field Definitions

\begin{tabular}{|c|c|c|}
\hline Field Name & Table & Description \\
\hline CALNOTE1 & CALIB & $\begin{array}{l}\text { Any comments which need recording about operation or } \\
\text { calibration of this instrument such as repairs made. }\end{array}$ \\
\hline CALNOTE2 & CALIB & Additional calibration notes. \\
\hline CATNO & PRODUCT & $\begin{array}{l}\text { Optional catalog number since it may not exist for all products. } \\
\text { May include alphabetical characters as well as numbers. }\end{array}$ \\
\hline CHEKORG & ORGANISE & $\begin{array}{l}\text { Implementation of a coding algorithm to enable searching for } \\
\text { "close" matches when importing tests. Since a given organization } \\
\text { may be entered into the database by multiple testing } \\
\text { laboratories, some scheme is necessary to find the closest match. } \\
\text { By offering the operator a small list of "close" matches, the } \\
\text { process of finding the matching wording in the target database is } \\
\text { simplified. }\end{array}$ \\
\hline CHEKPER & PEOPLE & $\begin{array}{l}\text { Implementation of a coding algorithm to enable searching for } \\
\text { "close" matches when importing tests. Since a given individual } \\
\text { may be entered into the database by multiple testing } \\
\text { laboratories, some scheme is necessary to find the closest match. } \\
\text { By offering the operator a small list of "close" matches, the } \\
\text { process of finding the matching wording in the target database is } \\
\text { simplified. }\end{array}$ \\
\hline CHEKPROD & PRODUCT & $\begin{array}{l}\text { Implementation of a coding algorithm to enable searching for } \\
\text { "close" matches when importing tests. Since a given product may } \\
\text { be entered into the database by multiple testing taboratories, } \\
\text { some scheme is necessary to find the closest match. By offering } \\
\text { the operator a small list of "close" matches, the process of } \\
\text { finding the matching wording in the target database is simplified. }\end{array}$ \\
\hline $\mathrm{CO} 180$ & CONE, FURN, ROOM & $\begin{array}{l}\text { Average CO yield over } 180 \text { seconds subsequent to ignition } \\
(\mathrm{kg} / \mathrm{kg}) \text {. }\end{array}$ \\
\hline $\mathrm{CO} 2180$ & CONE, FURN, ROOM & $\begin{array}{l}\text { Average } \mathrm{CO}_{2} \text { yield over } 180 \text { seconds subsequent to ignition } \\
(\mathrm{kg} / \mathrm{kg}) \text {. }\end{array}$ \\
\hline $\mathrm{CO} 2300$ & CONE, FURN, ROOM & $\begin{array}{l}\text { Average } \mathrm{CO}_{2} \text { yield over } 300 \text { seconds subsequent to ignition } \\
(\mathrm{kg} / \mathrm{kg}) \text {. }\end{array}$ \\
\hline $\mathrm{CO} 260$ & CONE, FURN, ROOM & $\begin{array}{l}\text { Average } \mathrm{CO}_{2} \text { yield over } 60 \text { seconds subsequent to ignition } \\
(\mathrm{kg} / \mathrm{kg}) \text {. }\end{array}$ \\
\hline $\mathrm{CO} 300$ & CONE, FURN, ROOM & $\begin{array}{l}\text { Average } \mathrm{CO} \text { yield over } 300 \text { seconds subsequent to ignition } \\
(\mathrm{kg} / \mathrm{kg}) \text {. }\end{array}$ \\
\hline $\mathrm{CO} 60$ & CONE, FURN, ROOM & $\begin{array}{l}\text { Average CO yield over } 60 \text { seconds subsequent to ignition } \\
(\mathrm{kg} / \mathrm{kg}) \text {. }\end{array}$ \\
\hline COMMDATE & INSTRUM & Date the instrument was first commissioned. \\
\hline COMMENT1 & CONE, FURN, LIFT, ROOM & $\begin{array}{l}\text { Test comments entered by the operator any time before, during, } \\
\text { or after a test. In some cases, e.g., second ignition, the comment } \\
\text { is directly inserted by the device software and not by the } \\
\text { operator. }\end{array}$ \\
\hline COMMENT2 & CONE, FURN, LIFT, ROOM & Additional operator comments. \\
\hline
\end{tabular}




\begin{tabular}{|c|c|c|}
\hline Field Name & Table & Description \\
\hline COMMENT3 & CONE, FURN, LIFT, ROOM & Additional operator comments. \\
\hline COMMENT4 & CONE, FURN, LIFT, ROOM & Additional operator comments. \\
\hline COMMENT5 & CONE, FURN, LIFT, ROOM & Additional operator comments. \\
\hline COMPOS & PRODUCT & Indicates if the product is a composite. \\
\hline CONSTO & CALIB & $\begin{array}{l}\text { Constant required for the conversion of instrument data to } \\
\text { physical units. Refer to the conversion equations in the CONV } \\
\text { field description for exact details. }\end{array}$ \\
\hline CONST1 & CALIB & $\begin{array}{l}\text { Constant required for the conversion of instrument data to } \\
\text { physical units. Refer to the conversion equations in the CONV } \\
\text { field description for exact details. }\end{array}$ \\
\hline CONST2 & CALIB & $\begin{array}{l}\text { Optional constant used for the polynomial conversion of } \\
\text { instrument data to physical units. Refer to the polynomial } \\
\text { equation in the CONV field description for exact details. }\end{array}$ \\
\hline CONST3 & CALIB & $\begin{array}{l}\text { Optional constant used for the polynomial conversion of } \\
\text { instrument data to physical units. Refer to the polynomial } \\
\text { equation in the CONV field description for exact details. }\end{array}$ \\
\hline CONST4 & CALIB & $\begin{array}{l}\text { Optional constant used for the polynomial conversion of } \\
\text { instrument data to physical units. Refer to the polynomial } \\
\text { equation in the CONV field description for exact details. }\end{array}$ \\
\hline CONST5 & CALIB & $\begin{array}{l}\text { Optional constant used for the polynomial conversion of } \\
\text { instrument data to physical units. Refer to the polynomial } \\
\text { equation in the CONV field description for exact details. }\end{array}$ \\
\hline CONTACID & PRODUCT & $\begin{array}{l}\text { PERSONID number from the PEOPLE table for the contact } \\
\text { within the manufacturing company. }\end{array}$ \\
\hline CONTACT & PRODUCT & $\begin{array}{l}\text { Name of a contact within the manufacturing company. The } \\
\text { name must match an individual in the PEOPLE table. }\end{array}$ \\
\hline
\end{tabular}




\begin{tabular}{|c|c|c|}
\hline Field Name & Table & Description \\
\hline CONV & CALIB & 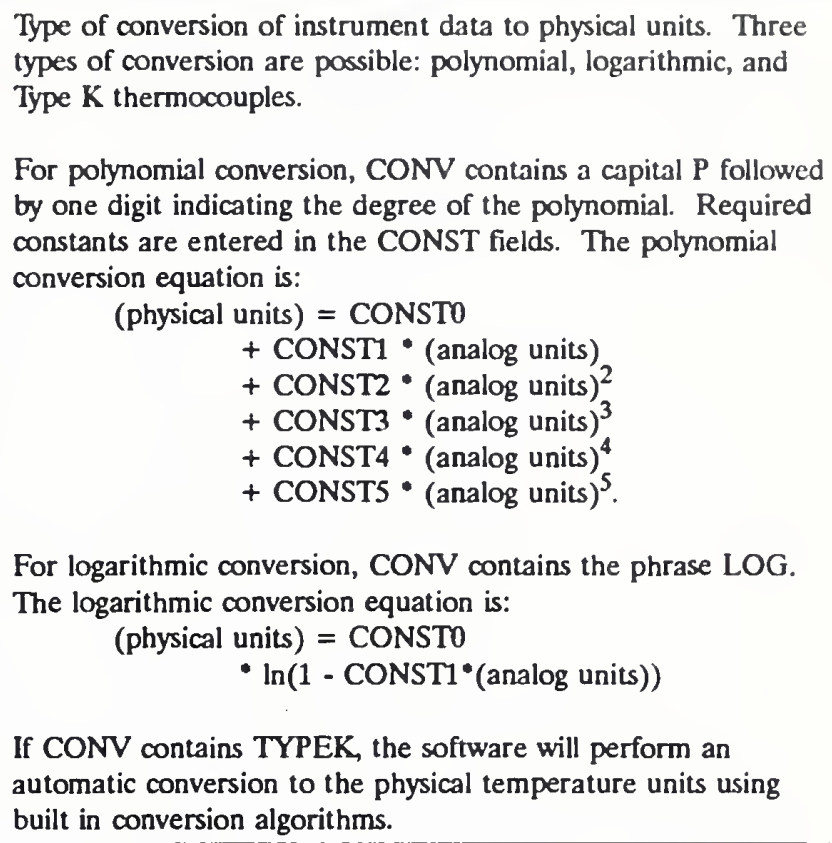 \\
\hline COUNTRY & ORGANISE, PEOPLE & $\begin{array}{l}\text { Country name, common name instead of full name (e.g., USA, } \\
\text { not United States of America) }\end{array}$ \\
\hline DELETED & ALL & Used by the database system to indicate a deleted record. \\
\hline DENSITY & CONE, FURN, LIFT, ROOM & Density of the composite product $\left(\mathrm{kg} / \mathrm{m}^{3}\right)$. \\
\hline DIVISION & ORGANISE, PEOPLE & $\begin{array}{l}\text { Division/department/branch (e.g., Building and Fire Research } \\
\text { Laboratory). }\end{array}$ \\
\hline E & CONE, FURN, ROOM & $\begin{array}{l}\text { Oxygen consumption constant. A generic value for this is } 13.1 \\
\mathrm{~kJ} / \mathrm{gO}_{2} \text {. If the composition of the fuel is known }\left(e . g ., \mathrm{CH}_{4} \text { or }\right. \\
\mathrm{PMMA} \text {, a more exact value can be used. For the Cone } \\
\text { Calorimeter, the data acquisition program lets the operator } \\
\text { specify the value to use from a menu at runtime. For instance, } \\
\text { for PMMA, this value would be } 12.98 \mathrm{~kJ} / \mathrm{gO}_{2} \text {. The data } \\
\text { reduction program uses the value in this field by default. }\end{array}$ \\
\hline FAX & ORGANISE, PEOPLE & Facsimile number, including country code. \\
\hline FILE & CONE, FURN, LIFT, ROOM & $\begin{array}{l}\text { Reserved for a laboratory-specific identification of the test series } \\
\text { to which the test belongs. This is typically a way to refer to the } \\
\text { sponsorship of a test. In addition to FILE, some laboratories } \\
\text { call this "Test Code," "Job Number," Test Reference,". }\end{array}$ \\
\hline FIRSTNAM & PEOPLE & First name of an individual. \\
\hline FLAMEOUT & CONE, FURN, LIFT, ROOM & $\begin{array}{l}\text { Time to flameout (s). This is the time of the last flameout if } \\
\text { more than one ignition/flameout has occurred. The remaining } \\
\text { values are recorded in the comments. }\end{array}$ \\
\hline FLASH & ROOM & Time (s) when flashover is observed in the room. \\
\hline
\end{tabular}




\begin{tabular}{|c|c|c|}
\hline Field Name & Table & Description \\
\hline FLOW & CONE, FURN, ROOM & Flow rate of a gas burner. \\
\hline FLUX & CONE, FURN, ROOM & Flux $\left(\mathrm{kW} / \mathrm{m}^{2}\right)$ \\
\hline FRAME & CONE & $\begin{array}{l}\text { Denotes if the edge frame was used (meaningful only for } \\
\text { horizontal orientations). }\end{array}$ \\
\hline FULLNAME & PEOPLE & $\begin{array}{l}\text { Composite name of an individual, e.g., both first and last names. } \\
\text { This is useful when it is necessary to reference a single field to } \\
\text { get a complete name rather than separate first and last name } \\
\text { fields. Separate fields are also included to provide easier sorting. }\end{array}$ \\
\hline GRID & CONE & Denotes if the wire grid was used. \\
\hline $\mathrm{H} 2 \mathrm{O} 180$ & CONE, FURN, ROOM & $\begin{array}{l}\text { Average } \mathrm{H}_{2} \mathrm{O} \text { yield over } 180 \text { seconds subsequent to ignition } \\
(\mathrm{kg} / \mathrm{kg}) \text {. }\end{array}$ \\
\hline $\mathrm{H} 2 \mathrm{O} 300$ & CONE, FURN, ROOM & $\begin{array}{l}\text { Average } \mathrm{H}_{2} \mathrm{O} \text { yield over } 300 \text { seconds subsequent to ignition } \\
(\mathrm{kg} / \mathrm{kg}) \text {. }\end{array}$ \\
\hline $\mathrm{H} 2 \mathrm{O} 60$ & CONE, FURN, ROOM & $\begin{array}{l}\text { Average } \mathrm{H}_{2} \mathrm{O} \text { yield over } 60 \text { seconds subsequent to ignition } \\
(\mathrm{kg} / \mathrm{kg}) \text {. }\end{array}$ \\
\hline HBR & CONE, FURN, ROOM & Similar to $\mathrm{HCL}$, but for $\mathrm{HBr}$. \\
\hline HC180 & CONE, FURN, ROOM & Average $\Delta h_{c}$ over 180 seconds subsequent to ignition $(\mathrm{kJ} / \mathrm{g})$. \\
\hline HC300 & CONE, FURN, ROOM & Average $\Delta h_{c}$ over 300 seconds subsequent to ignition $(\mathrm{kJ} / \mathrm{g})$. \\
\hline HC60 & CONE, FURN, ROOM & Average $\Delta h_{c}$ over 60 seconds subsequent to ignition $(\mathrm{kJ} / \mathrm{g})$ \\
\hline $\mathrm{HCL}$ & CONE, FURN, ROOM & $\begin{array}{l}\text { The yield of } \mathrm{HCl} \text {, as determined by batch analysis, typically by } \\
\text { ion chromatography. Similar types of measurement as the } \\
\text { SOOT field. These dimensionless quantities are determined } \\
\text { using the raw data (grams of species), the ratio of mass flow rate } \\
\text { through the solution to the main duct flow, and the mass of } \\
\text { specimen loss during the test. }\end{array}$ \\
\hline $\mathrm{HCN}$ & CONE, FURN, ROOM & Similar to $\mathrm{HCL}$, but for $\mathrm{HCN}$. \\
\hline $\mathrm{HF}$ & & See TUH. \\
\hline IGNITOR & ROOM & $\begin{array}{l}\text { Choices are: } \\
\text { Standard sand burner }(170 \mathrm{~mm} \times 170 \mathrm{~mm}) \\
\text { Alternative sand burner }(305 \mathrm{~mm} \times 305 \mathrm{~mm}) \\
\text { O-ther }\end{array}$ \\
\hline
\end{tabular}




\begin{tabular}{|c|c|c|}
\hline Field Name & Table & Description \\
\hline IGNTYPE & FURN & 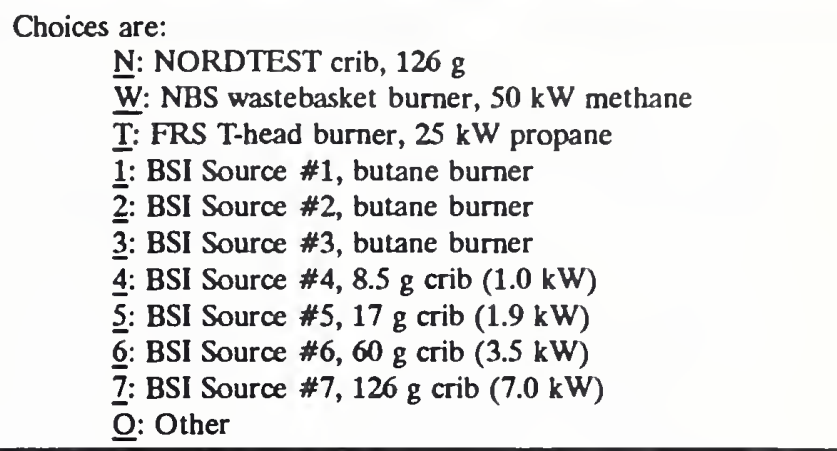 \\
\hline INERTIA & LIFT & Thermal inertia $\left(\mathrm{kW}^{2} \cdot \mathrm{s} \cdot \mathrm{m}^{-4} \cdot \mathrm{K}^{-2}\right)$ \\
\hline INITIAL & PEOPLE & $\begin{array}{l}\text { Middle initial for an individual. May include two letters, e.g., } \\
\text { Mc. }\end{array}$ \\
\hline INSTRID & INSTRUM & $\begin{array}{l}\text { Assigned by FDMS at the local site when a test is being } \\
\text { imported if the SERIAL field is not recognized as already } \\
\text { existing. It has no meaning outside of the local site. }\end{array}$ \\
\hline INSTRNO & CONE, FURN, LIFT, ROOM & $\begin{array}{l}\text { INSTRID number from the INSTRUM table to provide unique } \\
\text { identification for the test apparatus. Laboratories may have } \\
\text { more than one of a given type of fire test apparatus. }\end{array}$ \\
\hline INTERVAL & CONE, FURN, ROOM & Interval in seconds between two consecutive scans. \\
\hline LABID & CONE, FURN, LIFT, ROOM & $\begin{array}{l}\text { ORGID number from the ORGANISE table for the laboratory } \\
\text { where the test was conducted. }\end{array}$ \\
\hline LASTNAME & PEOPLE & The last name (surname, family name) of the individual. \\
\hline LOCATION & ROOM & $\begin{array}{l}\text { Location of the specimen. Choices are: } \\
\text { Corner of room } \\
\text { Wall center } \\
\text { O-ther }\end{array}$ \\
\hline MAINMAT & PRODUCT & $\begin{array}{l}\text { Generic name for the main material (e.g., rigid polyurethane } \\
\text { foam) composing a product. }\end{array}$ \\
\hline MAINUSE & PRODUCT & 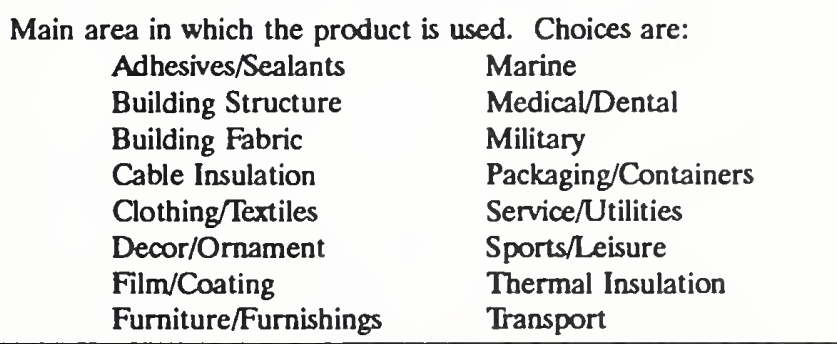 \\
\hline MAKER & INSTRUM & $\begin{array}{l}\text { Name of the company manufacturing this instrument. The name } \\
\text { must match an organization in the ORGANISE table. }\end{array}$ \\
\hline MAKERID & INSTRUM & $\begin{array}{l}\text { ORGID number from the ORGANISE table for the company } \\
\text { manufacturing this instrument. }\end{array}$ \\
\hline
\end{tabular}




\begin{tabular}{|c|c|c|}
\hline Field Name & Table. & Description \\
\hline MANUFACT & PRODUCT & $\begin{array}{l}\text { Name of the company manufacturing this product. The name } \\
\text { must exist in the ORGANISE table. }\end{array}$ \\
\hline MANUFID & PRODUCT & $\begin{array}{l}\text { ORGID number from the ORGANISE table for the company } \\
\text { manufacturing this product. }\end{array}$ \\
\hline MASSF & CONE, FURN, LIFT, ROOM & Specimen mass at the end of the test (g). \\
\hline MASSI & CONE, FURN, LIFT, ROOM & Specimen mass before the start of the test (g). \\
\hline MASSLOSS & CONE, FURN, LIFT, ROOM & Specimen mass loss during the test $(\mathrm{g})$. \\
\hline MAXEXT & ROOM & Maximum value of the smoke extinction area flow rate $\left(\mathrm{m}^{2} / \mathrm{s}\right)$. \\
\hline MAXMDOT & CONE, FURN, ROOM & $\begin{array}{l}\text { Peak mass loss rate } \mathrm{m}^{n}\left(\mathrm{~g} / \mathrm{sm}^{2}\right) \text {. The mass loss rate data is a } \\
\text { numerically obtained multi-point estimate of the derivative of } \\
\text { the mass loss. Consequently, this value has been smoothed to } \\
\text { some extent. }\end{array}$ \\
\hline MAXODOT & CONE, FURN, ROOM & $\begin{array}{l}\text { Peak rate of heat release } \mathrm{q}^{n}\left(\mathrm{~kW} / \mathrm{m}^{2}\right) \text {. For some materials (e.g., } \\
\text { charring materials), rate of heat release curves have more than } \\
\text { one peak. This entry represents the highest value peak for the } \\
\text { entire test. }\end{array}$ \\
\hline MAXSIGMA & CONE, FURN, ROOM & $\begin{array}{l}\text { Peak specific smoke extinction area } \sigma_{\mathrm{m}}\left(\mathrm{m}^{2} / \mathrm{kg}\right) \text {. As the raw } \sigma_{\mathrm{m}} \\
\text { records the actual turbulent fluctuations in the duct velocity, the } \\
\text { instantaneous values of the extinction coefficient } \mathrm{k} \text { have quite a } \\
\text { bit of fluctuation. Therefore, the computed specific extinction } \\
\text { area makes use of a smoothing algorithm. }\end{array}$ \\
\hline MAXTIME & CONE, FURN, ROOM & Time (s) to the peak rate of heat release in MAXQDOT field. \\
\hline MDOT180 & CONE, FURN, ROOM & $\begin{array}{l}\text { Average mass loss rate } \mathrm{t}^{\text {" }} \text { over } 180 \text { seconds subsequent to } \\
\text { ignition }\left(\mathrm{g} / \mathrm{s} \mathrm{m}^{2}\right) \text {. }\end{array}$ \\
\hline MDOT300 & CONE, FURN, ROOM & $\begin{array}{l}\text { Average mass loss rate } \mathrm{m}^{n} \text { over } 300 \text { seconds subsequent to } \\
\text { ignition }\left(\mathrm{g} / \mathrm{s} \mathrm{m}^{2}\right) \text {. }\end{array}$ \\
\hline MDOT60 & CONE, FURN, ROOM & $\begin{array}{l}\text { Average mass loss rate } \mathrm{m}^{n} \text { over } 60 \text { seconds subsequent to } \\
\text { ignition }\left(\mathrm{g} / \mathrm{s}^{2}\right) \text {. }\end{array}$ \\
\hline MORPHONE & PEOPLE & $\begin{array}{l}\text { Telephone extension or an alternative telephone number for an } \\
\text { individual. }\end{array}$ \\
\hline MOUNT1 & ROOM & $\begin{array}{l}\text { Specifies the means of mounting. For example, "Glued with } \\
\text { Brand X glue, } 2 \mathrm{~cm} \text { diameter globs, spaced at } 30 \mathrm{~cm} . "\end{array}$ \\
\hline MOUNT2 & ROOM & Continuation of MOUNT1. \\
\hline NEXTDATE & CALIB & Date of the next calibration. \\
\hline NOTES1 & INSTRUM & $\begin{array}{l}\text { Special comments about the current or past use of this } \\
\text { instrument. For example, "All data recorded between date X } \\
\text { and Y are suspect." }\end{array}$ \\
\hline NOTES2 & INSTRUM & Additional instrument comments. \\
\hline NOTES3 & INSTRUM & Additional instrument comments. \\
\hline
\end{tabular}




\begin{tabular}{|c|c|c|}
\hline Field Name & Table & Description \\
\hline NOTES4 & INSTRUM & Additional instrument comments. \\
\hline NOTES5 & INSTRUM & Additional instrument comments. \\
\hline OFFICER & $\begin{array}{l}\text { CALIB, CONE, FURN, LIFT, } \\
\text { ROOM }\end{array}$ & $\begin{array}{l}\text { The name of the laboratory officer responsible for a test. For } \\
\text { CALIB, the individual who has signature authority to issue a } \\
\text { calibration report. The name must match an individual in the } \\
\text { PEOPLE table. }\end{array}$ \\
\hline OFFID & $\begin{array}{l}\text { CALIB, CONE, FURN, LIFT, } \\
\text { ROOM }\end{array}$ & $\begin{array}{l}\text { PERSONID number from the PEOPLE table for the laboratory } \\
\text { officer responsible for a test. For CALIB, the individual who } \\
\text { has signature authority to issue a calibration report. }\end{array}$ \\
\hline OPERATOR & $\begin{array}{l}\text { CALIB, CONE, FURN, LIFT, } \\
\text { ROOM }\end{array}$ & $\begin{array}{l}\text { The name of the person who performed the test. For CALIB, } \\
\text { the individual who actually performed the calibration. The name } \\
\text { must match an individual in the PEOPLE table. }\end{array}$ \\
\hline OPERID & $\begin{array}{l}\text { CALIB, CONE, FURN, LIFT, } \\
\text { ROOM }\end{array}$ & $\begin{array}{l}\text { PERSONID number from the PEOPLE table for the individual } \\
\text { who performed the test. For CALIB, the individual who } \\
\text { actually performed the calibration. }\end{array}$ \\
\hline ORGANISE & ORGANISE, PEOPLE & $\begin{array}{l}\text { Name of the organization (e.g., National Institute of Standards } \\
\text { and Technology). }\end{array}$ \\
\hline ORGDATE & ORGANISE & $\begin{array}{l}\text { When several sources of information are available for the same } \\
\text { organization, it may not be clear which information is the most } \\
\text { current. This field is updated only when it is known that the } \\
\text { information in the record is current and correct. If any } \\
\text { information being entered into an ORGANISE record is } \\
\text { uncertain, this field should be left blank. Such a version of the } \\
\text { record is preferentially discarded when a verified record becomes } \\
\text { available. }\end{array}$ \\
\hline ORGID & ORGANISE & Assigned to uniquely identify the organization. \\
\hline ORIENT & CONE & Specimen orientation, horizontal or vertical. \\
\hline OXYGEN & CONE, FURN, ROOM & $\begin{array}{l}\text { Nominal value of the oxygen concentration in the enclosure } \\
\text { around the heater and sample. The purpose is to enable quick } \\
\text { searching of the database. For tests run at non-ambient oxygen } \\
\text { concentration, the user may have installed a second oxygen } \\
\text { meter to monitor the concentration of the inflow. Such data are } \\
\text { recorded in a vector data channel. A typical value is } 20.95 \% \text {. }\end{array}$ \\
\hline PERDATE & PEOPLE & $\begin{array}{l}\text { When several sources of information are available for the same } \\
\text { individual, it may not be clear which information is the most } \\
\text { current. This field is updated only when it is known that the } \\
\text { information in the record is current and correct. If any } \\
\text { information being entered into a PEOPLE record is uncertain, } \\
\text { this field should be left blank. Such a version of the record is } \\
\text { preferentially discarded when a verified record becomes } \\
\text { available. }\end{array}$ \\
\hline PERSONID & PEOPLE & $\begin{array}{l}\text { Assigned to uniquely identify an individual. This is necessary to } \\
\text { distinguish two people with the same name. }\end{array}$ \\
\hline PHI & LIFT & Flame heating parameter $\left(\mathrm{kW}^{2} / \mathrm{m}^{3}\right)$. \\
\hline
\end{tabular}




\section{A Programmer's Reference Guide to FDMS File Formats}

\begin{tabular}{|c|c|c|}
\hline Field Name & Table & Description \\
\hline PHONE & ORGANISE, PEOPLE & Telephone, including country code. \\
\hline PILOT & CONE & Indicates if ignition was piloted.. \\
\hline POSTCODE & ORGANISE, PEOPLE & Postal code (Zip code for USA). \\
\hline PRDATE & PRODUCT & $\begin{array}{l}\text { When several sources of information are available for the same } \\
\text { product, it may not be clear which information is the most } \\
\text { current. This field is updated only when it is known that the } \\
\text { information in the record is current and correct. If any } \\
\text { information being entered into a PRODUCT record is } \\
\text { uncertain, this field should be left blank. Such a version of the } \\
\text { record is preferentially discarded when a verified record becomes } \\
\text { available. }\end{array}$ \\
\hline PRIVATE & CONE, FURN, LIFT, ROOM & $\begin{array}{l}\text { Allows a laboratory to define the level of access by other } \\
\text { organizations to test results in the database. Choices are: } \\
\text { - Allow the data to be exported without allowing } \\
\text { modifications. } \\
\text { - Purge any test information which might identify the } \\
\text { manufacturer before allowing export. } \\
\text { - Do not allow export under any circumstances. }\end{array}$ \\
\hline PRODENSI & PRODUCT & Density of the product $\left(\mathrm{kg} / \mathrm{m}^{3}\right)$. \\
\hline PRODESC1 & PRODUCT & Product description. \\
\hline PRODESC2 & PRODUCT & Continuation of product description. \\
\hline PRODESC3 & PRODUCT & Continuation of product description. \\
\hline PRODESC4 & PRODUCT & Continuation of product description. \\
\hline PRODESC5 & PRODUCT & Continuation of product description. \\
\hline PRODID & PRODUCT & $\begin{array}{l}\text { Assigned to uniquely identify the test product. This is necessary } \\
\text { since many products have similar names which are difficult to } \\
\text { distinguish. }\end{array}$ \\
\hline PRODID1 & CONE, FURN, LIFT, ROOM & $\begin{array}{l}\text { PRODID value from PRODUCT table for the main product } \\
\text { composing the sample. }\end{array}$ \\
\hline PRODID2 & CONE, FURN, LIFT, ROOM & $\begin{array}{l}\text { PRODID value from PRODUCT table for the secondary } \\
\text { product composing the sample. }\end{array}$ \\
\hline PRODNAME & PRODUCT & Commercial name of the test product. \\
\hline PRODUCT1 & CONE, FURN, LIFT, ROOM & $\begin{array}{l}\text { The name of the main product composing the sample. The } \\
\text { name must match a product in the PRODUCT table. }\end{array}$ \\
\hline PRODUCT2 & CONE, FURN, LIFT, ROOM & $\begin{array}{l}\text { The name of the sccondary product composing the sample. The } \\
\text { name must match a product in the PRODUCT table. }\end{array}$ \\
\hline PROTHICK & PRODUCT & Product thickness (mm). \\
\hline QDOT180 & CONE, FURN, ROOM & $\begin{array}{l}\text { Average rate of heat release } q^{n} \text { over } 180 \text { seconds subsequent to } \\
\text { ignition }\left(\mathrm{kW} / \mathrm{m}^{2}\right) \text {. }\end{array}$ \\
\hline
\end{tabular}




\begin{tabular}{|c|c|c|}
\hline Field Name & Table & Description \\
\hline QDOT300 & CONE, FURN, ROOM & $\begin{array}{l}\text { Average rate of heat release } q^{\prime \prime} \text { over } 300 \text { seconds subsequent to } \\
\text { ignition }\left(\mathrm{kW} / \mathrm{m}^{2}\right) \text {. }\end{array}$ \\
\hline QDOT60 & CONE, FURN, ROOM & $\begin{array}{l}\text { Average rate of heat release } q^{n} \text { over } 60 \text { seconds subsequent to } \\
\text { ignition }\left(\mathrm{kW} / \mathrm{m}^{2}\right) \text {. }\end{array}$ \\
\hline QIG & LIFT & Minimum flux for ignition $\left(\mathrm{kW} / \mathrm{m}^{2}\right)$. \\
\hline QSMIN & LIFT & Minimum flux for spread $\left(\mathrm{kW} / \mathrm{m}^{2}\right)$. \\
\hline RECEIVED & CONE, FURN, LIFT, ROOM & Date test results were received. \\
\hline REGION & ORGANISE, PEOPLE & State for USA, county for UK, etc. \\
\hline REPDATE & CONE, FURN, LIFT, ROOM & Date the test was reported. \\
\hline RHCOND & CONE, FURN, LIFT, ROOM & $\begin{array}{l}\text { Relative humidity for specimen conditioning (\%). This is } \\
\text { important if, for example, the specimens were oven-dried at } \\
\mathrm{RH}=0 \text {. }\end{array}$ \\
\hline RHTEST & CONE, FURN, LIFT, ROOM & $\begin{array}{l}\text { Relative humidity of the supply air for conducting the test (\%). } \\
\text { In the case of special, controlled atmospheres, this can be user } \\
\text { selected. }\end{array}$ \\
\hline SCANS & CONE, FURN, ROOM & $\begin{array}{l}\text { Total number of scans for the test. For Cone Calorimeter, value } \\
\text { is entered by CONERUN. }\end{array}$ \\
\hline SERIAL & INSTRUM & $\begin{array}{l}\text { Identical to the header line "SERIAL NAME" imported as part } \\
\text { of the raw data. }\end{array}$ \\
\hline SIGMA180 & CONE, FURN, ROOM & Average $\sigma_{\mathrm{m}}$ over 180 seconds subsequent to ignition $\left(\mathrm{m}^{2} / \mathrm{kg}\right)$. \\
\hline SIGMA300 & CONE, FURN, ROOM & Average $\sigma_{\mathrm{m}}$ over 300 seconds subsequent to ignition $\left(\mathrm{m}^{2} / \mathrm{kg}\right)$. \\
\hline SIGMA60 & CONE, FURN, ROOM & Average $\sigma_{\mathrm{m}}$ over 60 seconds subsequent to ignition $\left(\mathrm{m}^{2} / \mathrm{kg}\right)$. \\
\hline SOOT & CONE, FURN, ROOM & $\begin{array}{l}\text { Mass of the soot deposited on the soot filter during the test } \\
\text { divided by the mass of specimen loss during the test. }\end{array}$ \\
\hline SPCONTID & CONE, FURN, LIFT, ROOM & $\begin{array}{l}\text { PERSONID number from the PEOPLE table for the contact } \\
\text { person at the sponsoring organization. }\end{array}$ \\
\hline SPDATE1 & CONE, FURN, LIFT, ROOM & Supply date for product 1 . \\
\hline SPDATE2 & CONE, FURN, LIFT, ROOM & Supply date for product 2 . \\
\hline SPONCONT & CONE, FURN, LIFT, ROOM & $\begin{array}{l}\text { Name of the contact person at the sponsoring organization. This } \\
\text { name must match an individual in the PEOPLE table. }\end{array}$ \\
\hline SPONID & CONE, FURN, LIFT, ROOM & $\begin{array}{l}\text { ORGID number from the ORGANISE table for the sponsoring } \\
\text { organization. }\end{array}$ \\
\hline SPONSOR & CONE, FURN, LIFT, ROOM & $\begin{array}{l}\text { Name of the sponsoring organization. This name must match an } \\
\text { organization in the ORGANISE table. }\end{array}$ \\
\hline SUMEXT & ROOM & Total smoke extinction area released during the entire test $\left(\mathrm{m}^{2}\right)$. \\
\hline
\end{tabular}




\begin{tabular}{|c|c|c|}
\hline Field Name & Table & Description \\
\hline SURFDENS & ROOM & $\begin{array}{l}\text { When thin textiles, papers, etc., are covering some standard } \\
\text { substrate in a ROOM test, it is most appropriate to describe } \\
\text { them by their surface density }\left(\mathrm{kg} / \mathrm{m}^{2}\right) \text {. }\end{array}$ \\
\hline TELEX & ORGANISE, PEOPLE & Telex number. \\
\hline TEMPCOND & CONE, FURN, LIFT, ROOM & Temperature $\left({ }^{\circ} \mathrm{C}\right)$ for specimen conditioning. \\
\hline TEMPTEST & CONE, FURN, LIFT, ROOM & Temperature $\left({ }^{\circ} \mathrm{C}\right)$ of the supply air for conducting the test. \\
\hline TEST & CONE, FURN, LIFT, ROOM & $\begin{array}{l}\text { Serial test number assigned. It is specific to the laboratory and } \\
\text { to an instrument. For the Cone Calorimeter, it is assigned by } \\
\text { the CONERUN software. }\end{array}$ \\
\hline TESTDATE & CONE, FURN, LIFT, ROOM & Date the original test was run. \\
\hline THICK & CONE, FURN, LIFT, ROOM & Specimen thickness $(\mathrm{m})$ \\
\hline TIG & LIFT & Minimum temperature for ignition $\left({ }^{\circ} \mathrm{C}\right)$. \\
\hline TIGN & CONE, FURN, LIFT, ROOM & $\begin{array}{l}\text { Time to ignition, defined as sustained flaming (s). This is the } \\
\text { time of first ignition if more than one ignition/lameout has } \\
\text { occurred. The remaining values are recorded with the } \\
\text { comments. }\end{array}$ \\
\hline TOTLHEAT & CONE, FURN, ROOM & Total heat released during the entire test (MJ). \\
\hline TSMIN & LIFT & Minimum temperature for spread $\left({ }^{\circ} \mathrm{C}\right)$. \\
\hline TSTAR & LIFT & Characteristic equilibrium or thermal steady state time (s). \\
\hline TUH & CONE, FURN, ROOM & $\begin{array}{l}\text { Similar to HCL, but for total unburned fuel. The HF field } \\
\text { originally allocated to hydrogen fluoride measurements was } \\
\text { reallocated to total unburned hydrocarbons, TUH, to conform } \\
\text { to current usage in laboratories. }\end{array}$ \\
\hline USERIS & CONE, FURN, LIFT, ROOM & Additional user defined strings. \\
\hline USER2S & CONE, FURN, LIFT, ROOM & $\begin{array}{l}\text { Contains user defined strings. This could be a variable name } \\
\text { identifying the value in one of the user defined numeric fields: } \\
\text { USERNUM1, USERNUM2, USERNUM3. Information } \\
\text { entered is site-specific. It is not exported by the FDMS export } \\
\text { module since it has no meaning in other implementations of } \\
\text { FDMS. }\end{array}$ \\
\hline USER3\$ & CONE, FURN, LIFT, ROOM & Additional user defined strings. \\
\hline USERNUM1 & CONE, FURN, LIFT, ROOM & $\begin{array}{l}\text { Contains user defined numeric data. For example, for a given } \\
\text { test series the yield of } \mathrm{NO}_{\mathrm{x}} \text { may be one of the measurements. } \\
\text { The user could attribute this field to the test-average } \mathrm{NO}_{\mathrm{x}} \text { yield. } \\
\text { Information entered is site-specific. It is not exported by the } \\
\text { FDMS export module since it has no meaning in other } \\
\text { implementations of FDMS. }\end{array}$ \\
\hline USERNUM2 & CONE, FURN, LIFT, ROOM & Additional user defined numeric data. \\
\hline USERNUM3 & CONE, FURN, LIFT, ROOM & Additional user defined numeric data. \\
\hline
\end{tabular}


Field Definitions

\begin{tabular}{|l|l|l|}
\hline Field Name & Table & Description \\
\hline VERSION & CONE, FURN, LIFT, ROOM & $\begin{array}{l}\text { FDMS version number. Required to identify the correct version } \\
\text { of the data reduction routines. }\end{array}$ \\
\hline ZNUMBER & CONE, FURN, LIFT, ROOM & $\begin{array}{l}\text { Mechanism by which the DOS vector data file is associated with } \\
\text { a specific test. The name of the DOS file is the ZNUMBER } \\
\text { with a prefix of "Z". }\end{array}$ \\
\hline
\end{tabular}


7. References

[1] Babrauskas, V., Peacock, R.D., Janssens, M., and Batho, N.E., Standardizing the Exchange of Fire Data - The FDMS, Fire and Materials 15, 85-92 (1991).

[2] Beta version software distributed by Fire Research Station, Borehamwood, England, attn: S.A. Ames.

[3] Babrauskas, V., Janssens, M., Peacock, R.D., and Batho, N.E., Technical Documentation and User's Guide for FDMS, A Fire Data Management System, unpublished (1990).

[4] Dark Star Research Ltd., Penley, Clwyd, England. 


